\title{
Propagation of Damage in Brain Tissue: Coupling the Mechanics of Edema and Oxygen Delivery
}

\author{
Georgina E. Lang • Dominic Vella · Sarah L. Waters · Alain \\ Goriely
}

Received: date / Accepted: date

\begin{abstract}
Brain tissue swelling, or edema, is a dangerous consequence of traumatic brain injury and stroke. In particular, a locally swollen region can cause the injury to propagate further through the brain: swelling causes mechanical compression of the vasculature in the surrounding tissue and so can cut off that tissue's oxygen supply. We use a triphasic mathematical model to investigate this propagation, and couple tissue mechanics with oxygen delivery. Starting from a fully coupled, finite elasticity, model, we show that simplifications can be made that allow us to express the volume of the propagating region of damage analytically in terms of key parameters. Our results show that performing a craniectomy, to alleviate pressure in the brain and allow the tissue to swell outwards, reduces the propagation of damage; this finding agrees with experimental observations.
\end{abstract}

Keywords Oxygen delivery · triphasic model · brain mechanics · mathematical modelling · tissue swelling

\section{Introduction}

Following damage such as a stroke or traumatic brain injury, brain tissue has been observed to swell (Simard et al., 2007). Brain swelling, or edema, is a significant cause of morbidity and death (Raslan and Bhardwaj, 2007); current medical strategies aim to reduce swelling and intracranial pressure to maintain blood supply and retain cerebral metabolism (Thiex and Tsirka, 2007). However, to enable treatments to be developed it is of critical importance to understand the underlying physical effects that cause brain tissue to swell following injury (Goriely et al., 2015).

In healthy brain tissue the delicate balance of ions is maintained by active ion pumping. This prevents excess movement of water into tissue in response to osmotic pressures (Simard et al., 2007). However, when tissue is damaged or dies this balance is disturbed leading to the movement of water and, hence, edema. Localized edema can cause further tissue damage to propagate through healthy brain tissue (Gerriets et al., 2004). This propagation occurs because the swollen tissue occupies additional space within the brain resulting in mechanical deformation, compression of blood vessels and high intracranial pressure (ICP) in the previously healthy tissue surrounding the swollen region. This surrounding tissue subsequently suffers from a lack of blood flow (ischemia), which leads to hypoxia and, ultimately, to cell death (Flechsenhar et al., 2013). Cell death can, in turn, lead to the propagation of further swelling and damage. A schematic of this process is shown in Figure 1.

To break the vicious cycle of propagation of tissue damage, two medical interventions are common: treatment with osmotic agents (such as Mannitol, see Papadopoulos et al., 2002, for example) and craniectomy (the removal of a portion of the skull). Treatment with osmotic agents is not always possible, for example if the blood-brain barrier has become compromised. Here we focus on craniectomy, which is known to reduce high pressure in the brain, and improve blood flow in a swollen brain (Cooper et al., 2011). The idea is that the brain is allowed to increase in volume so that the adverse compression caused by swelling can be (partially) removed. The experiments of Walberer et al. (2008) highlighted the consequences of the space occupying effect of edema, by comparing the spread of edema in rats with a craniectomy to those with an intact skull. A stroke was induced in one hemisphere, and the volume of infarcted (dead) tissue and midline shift were measured after 5 and 24 hours (the midline shift is the deviation of the center line of the brain during swelling). At both time points, the rats that had undergone

G. E. Lang, D. Vella, S. L. Waters, A. Goriely

Mathematical Institute, University of Oxford

E-mail: goriely@maths.ox.ac.uk 
craniectomy showed less midline shift, and had a smaller volume of infarcted tissue than those with an intact skull. The authors suggest that the craniectomy contributed to a better outcome because the brain tissue could swell outwards, preventing the regional compression of blood vessels to the area surrounding the originally infarcted region. However, craniectomy is also associated with negative outcomes because of the additional stretching, and possible damage, of axons (Cooper et al., 2011).

We propose a mathematical model to investigate the propagation of damage through the brain. In particular, we focus on whether changing the boundary conditions at the outer edge of the domain (representing either the intact skull or a craniectomy) affects how far damage propagates through the brain.

\section{Mathematical modelling approach}

Brain cells contain an intracellular fixed charge density (FCD), comprised of negatively charged macromolecules, which contributes to brain edema (Elkin et al., 2010). In healthy tissue, this FCD is isolated from the tissue interstitium because the cell membranes actively pump ions to maintain osmotic equilibrium. However, when brain tissue is damaged or receives insufficient oxygen, the cells can no longer support active pumping and this FCD is exposed. This exposed FCD induces an ion concentration difference between the damaged tissue and its surroundings (the Donnan effect), resulting in an osmotic pressure difference, water accumulation, and hence swelling (Elkin et al., 2010; Lang et al., 2014).

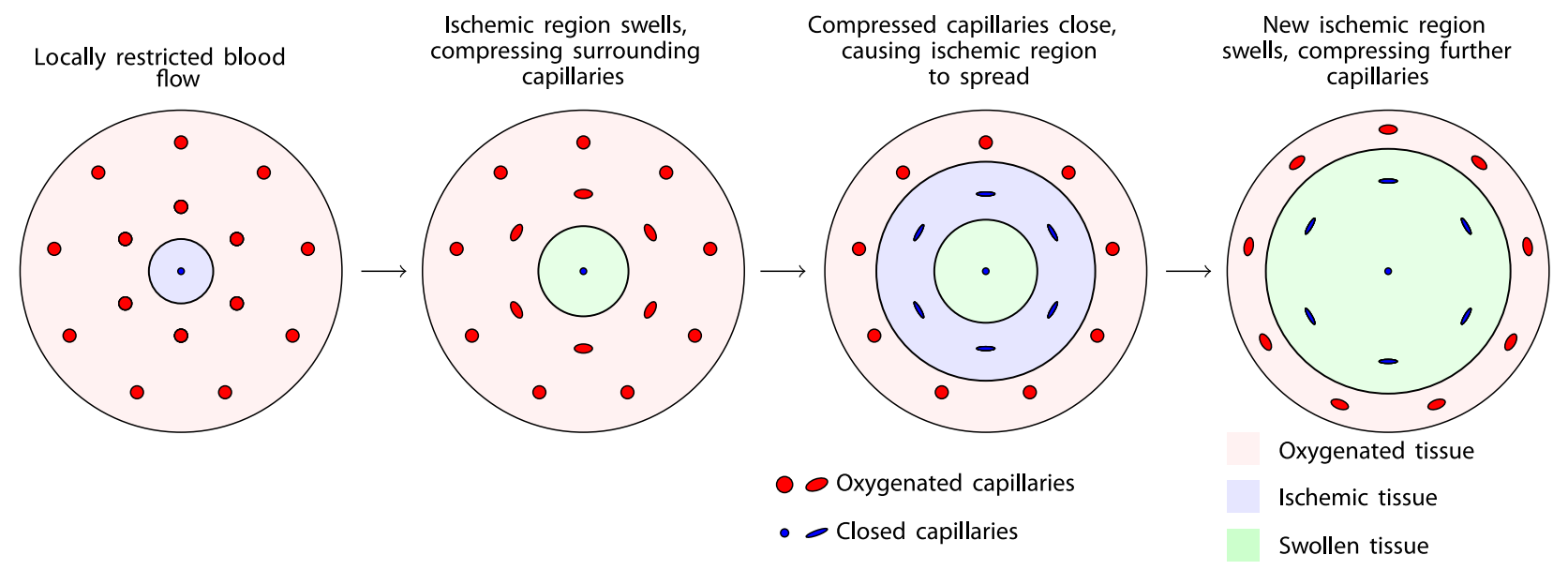

Fig. 1 Schematic of the mechanism by which localized ischemia can propagate throughout the brain.

We incorporate the exposure of intracellular FCD into a model that couples the deformation of, and oxygen distribution through, a region of tissue. The tissue will be modelled as a triphasic mixture (consisting of a charged solid, fluid, and positive and negative ion phases) together with a diffusion-uptake model for the oxygen concentration within the tissue. Capillaries are located throughout the tissue, and provide a source of oxygen when they are open. We model the effect of tissue deformation on the oxygen supply by assuming that capillaries close when they experience a compressive stress above a critical threshold. The oxygen distribution provides a feedback into the tissue mechanics, as we assume that the tissue becomes infarcted and develops an FCD wherever the oxygen concentration falls below a threshold value.

We shall use an equilibrium model to describe both the tissue mechanics and oxygen distribution. This assumption is appropriate because calculations show that the timescales for water, ion, and oxygen movement through the tissue are significantly shorter than the timescale on which the evolution of edema is observed experimentally (Lang, 2014). Edema is observed to develop over the duration of days (Hatashita et al., 1988; Marmarou et al., 2000) whilst, for example, the timescale of ion movement through the brain is $H^{2} / D \approx 2$ hours, where $D=10^{-10} \mathrm{~m}^{2} \mathrm{~s}^{-1}$ is a typical diffusion coefficient for ions in soft tissue (Sun et al., 1999) and $H=1 \mathrm{~mm}$ is a typical length scale. This estimate suggests that it is the rate of tissue damage, for example the rate at which ischemic tissue dies and exposes the intracellular FCD, rather than mechanical effects (such as the rate of interstitial fluid movement, or oxygen diffusion), that governs the rate of evolution of edema.

To examine the propagation of damage (and hence edema), we employ an iterative model: at stage $n$, we compute the region within which the capillaries are expected to be compressed. Subsequently, in stage $n+1$, we 
close these capillaries, and find the new infarcted region within which the oxygen concentration has fallen below the threshold. We then calculate the stresses in the tissue that would be caused by this new FCD distribution. This iteration process is discussed in more detail in $\S 2.5$. Beforehand, in $\S 2.1$, we discuss the model geometry that we employ, while in $\S 2.2$ and $\S 2.3$ we give the governing equations for an equilibrium triphasic model and diffusion-uptake of oxygen on a deformable domain.

\subsection{Geometry}

We consider a spherically symmetric region of tissue, of outer radius $H$. This is the simplest geometry that is able to reproduce the spatial variations in compressive stress that are observed in the brain. We assume that capillaries are evenly spaced throughout the tissue a distance $H_{\text {cap }}$ apart. Hence there are $N=H / H_{\text {cap }}$ capillaries in the tissue, with the first capillary at radius $R=H_{\text {cap }} / 2$, and the $i$ th capillary at radius $R_{\text {cap }}^{(i)}=(i-1 / 2) H_{\text {cap }}$ $(i=1 \ldots N)$. We will not model the mechanical behaviour of the capillaries, but rather treat each capillary as a point source of oxygen. We note that the spherically symmetric geometry means that the capillaries are actually 'shells' of capillary rather than individual vessels.

\subsection{Mechanics}

The tissue is bathed in a fluid of ionic concentration $c^{*}$, with reference pressure $p=0$, representing the cerebrospinal fluid (CSF) that surrounds the brain (the CSF pressure is not assumed to vanish, rather we take it as a reference pressure in computing pressure differences). The tissue is described in the Lagrangian (reference) configuration by the material coordinates $\mathbf{X}=R \hat{\mathbf{e}}_{r}$, and in the Eulerian (current) configuration by $\mathbf{x}=r \hat{\mathbf{e}}_{r}$, where $\hat{\mathbf{e}}_{r}$ is a unit normal in the radial direction. To investigate the mechanical deformation of the tissue, we seek a spherically symmetric deformation of the form $r=f(R)$. Then the deformation gradient may be written in spherical coordinates as

$$
\mathbf{F}=\nabla_{\mathbf{X}} \mathbf{x}(\mathbf{X})=\left(\begin{array}{ccc}
f^{\prime} & 0 & 0 \\
0 & \frac{f}{R} & 0 \\
0 & 0 & \frac{f}{R}
\end{array}\right), \quad J=\operatorname{det}(\mathbf{F})=\frac{f^{2} f^{\prime}}{R^{2}}
$$

(Ben Amar and Goriely, 2005) where' denotes differentiation with respect to $R$.

We assume that the tissue consists of a triphasic mixture with a charged solid phase, fluid phase, and positive and negative ion species in solution. At each iteration the reference state $\mathrm{FCD}, c_{0}^{(f)}(R)$, will be determined from the steady state oxygen distribution given a certain level of damage (see $\S 2.5$ ).

The governing equations for a triphasic mixture at equilibrium are discussed by, for example, Lai et al. (1991); Huyghe and Janssen (1997); Lang et al. (2014), and we summarise them here. The Cauchy stress is comprised of two parts, the effective stress $\boldsymbol{\sigma}_{e}$ and the pore pressure $p$,

$$
\boldsymbol{\sigma}=\boldsymbol{\sigma}_{e}-p \mathbf{I}
$$

where the effective stress, $\boldsymbol{\sigma}_{e}$, is related to a strain energy function $W(\boldsymbol{F})$ by

$$
\boldsymbol{\sigma}_{e}=\frac{1}{J} \boldsymbol{F} \cdot \frac{\partial W}{\partial \boldsymbol{F}}
$$

It was shown by Lang et al. (2014) that for volume changes of less than $50 \%$, different choices of isotropic materials lead to negligible differences in stresses. We therefore adopt a simple neo-Hookean model here; details are given in Appendix A. The stress balance reads,

$$
\nabla \cdot \boldsymbol{\sigma}_{e}=\nabla p
$$

As already discussed, we assume that the mixture is at equilibrium; in particular, there is no fluid flux through the tissue. Therefore, assuming electroneutrality and a dilute solutions, the chemical potential of solvent is constant and the pore pressure is proportional to the osmotic pressure,

$$
p=R T\left[\left(c^{+}+c^{-}\right)-c^{*}\right],
$$


where $R$ is the ideal gas constant, $T$ the absolute temperature, and $c^{+}, c^{-}, c^{*}$ are the concentrations of positive and negative ions in the tissue, and the bathing solution, respectively. At equilibrium the concentrations $c^{+}, c^{-}$ are determined by the Donnan equilibrium,

$$
c^{+}+c^{-}=\left[c^{* 2}+c^{(f)^{2}}\right]^{1 / 2}
$$

where $c^{(f)}$ is the FCD (Donnan, 1924; Cowin and Doty, 2009). As this FCD is attached to the solid phase, the FCD is dependent upon the deformation of the solid phase,

$$
c^{(f)}=\frac{\phi_{0}^{w} c_{0}^{(f)}(R)}{J-1+\phi_{0}^{w}}
$$

where $\phi_{0}^{w}$ is the brain tissue water content in the reference state, that is the overall volume fraction of water in the brain that is relevant for ion/FCD concentrations, and $c_{0}^{(f)}(R)$ is the reference state FCD.

We now eliminate $p, c^{(f)}, c^{+}$and $c^{-}$from equations (4)-(7) to yield a single differential equation for the deformation $f(R)$. Combining equations (4)-(6), we find that the divergence of the effective stress tensor balances the gradient of the osmotic pressure,

$$
\nabla \cdot \boldsymbol{\sigma}_{e}=R T \nabla\left(c^{+}+c^{-}\right) .
$$

Next the solute concentrations may be replaced by substituting in the Donnan equilibrium (6), and mass conservation equation for the $\mathrm{FCD}(7)$,

$$
\nabla \cdot \boldsymbol{\sigma}_{e}=R T \nabla\left(\left[c^{* 2}+\left(\frac{\phi_{0}^{w} c_{0}^{(f)}(R)}{J-1+\phi_{0}^{w}}\right)^{2}\right]^{1 / 2}\right) .
$$

We observe that for a given strain energy function $W(\mathbf{F})$, the effective stress $\boldsymbol{\sigma}_{e}$ and dilation $J$ are both functions of the deformation through equations (1) and (3). Thus equations (4)-(7) are replaced by a single equation for $f(R)$ governing the displacement of the system.

Equation (9) contains derivatives in the Eulerian configuration. We map the equations into the Lagrangian configuration so that the system may be solved on the (fixed) reference domain $R \in[0, H]$ rather than the deformed current domain. The stress balance may be reformulated into a Lagrangian frame momentum equation so that,

$$
\frac{\nabla_{\mathbf{X}} \cdot \mathbf{S}_{e}}{R T}=\nabla_{\mathbf{X}} \cdot\left(J \mathbf{F}^{-\mathrm{T}}\left[c^{* 2}+\left(\frac{\phi_{0}^{w} c_{0}^{(f)}(R)}{J-1+\phi_{0}^{w}}\right)^{2}\right]^{1 / 2}\right)
$$

where $\mathbf{S}_{e}$ is the effective first Piola-Kirchhoff stress tensor, related to the effective Cauchy stress by $\boldsymbol{\sigma}_{e}=(1 / J) \mathbf{S}_{e}$. $\mathbf{F}^{\mathrm{T}}$. Note that $\mathbf{F}^{-\mathrm{T}}$ stands for the transpose of the inverse of $\mathbf{F}$.

To close the problem, we must specify suitable boundary conditions for the displacement, $f(R)$. At $R=0$, we impose no displacement. On the outer boundary, $R=H$, we impose either no displacement (representing an intact skull) or free swelling (representing a craniectomy). We therefore have

$$
\left.f\right|_{0}=0, \quad \begin{cases}\left.f\right|_{H}=H, & \text { (intact skull) } \\ \left.\hat{\mathbf{e}}_{r} \cdot \boldsymbol{\sigma}\right|_{H} \cdot \hat{\mathbf{e}}_{r}=0, & \text { (craniectomy) }\end{cases}
$$

where

$$
\boldsymbol{\sigma}=\boldsymbol{\sigma}_{e}-R T\left(\left[c^{* 2}+\left(\frac{\phi_{0}^{w} c_{0}^{(f)}(R)}{J-1+\phi_{0}^{w}}\right)^{2}\right]^{1 / 2}-c^{*}\right)
$$

is the Cauchy stress.

Once the deformation $f(R)$ has been determined, the stress $\boldsymbol{\sigma}(R)$ is known everywhere (from equations (1), (3) and (12)). We must now determine the damage. To do so, we define a critical stress, $\sigma_{\text {crit }}$, the compressive stress at which we assume capillaries are compressed and cease to function. If the maximum compressive stress at a position $R$ within the tissue, $\sigma_{\max }(R)$, exceeds $\sigma_{\text {crit }}$ then the tissue at that location becomes ischemic and hence damaged. We will fix $\sigma_{\text {crit }}$ as a constant parameter; this assumption means that we neglect the autoregulatory 
mechanisms of the brain, whereby arteries dilate in response to increased intracranial pressure in order to maintain blood flow (Steiner and Andrews, 2006).

Since we are considering a spherically symmetric geometry, $\sigma_{\max }(R)$ is equal to the maximum of the radial and circumferential components of the compressive Cauchy stress,

$$
\sigma_{\max }(R):=\max \left\{-\left(\hat{\mathbf{e}}_{r} \cdot \boldsymbol{\sigma} \cdot \hat{\mathbf{e}}_{r}\right),-\left(\hat{\mathbf{e}}_{\theta} \cdot \boldsymbol{\sigma} \cdot \hat{\mathbf{e}}_{\theta}\right)\right\}
$$

Note that in the definition of the Cauchy stress, $\boldsymbol{\sigma}=\boldsymbol{\sigma}_{e}-p \mathbf{I}$, a positive stress is tensile. We have therefore taken the maximum of the negative components of the stress to determine the maximum compressive stress in (13).

\subsection{Oxygen}

We now consider the transport of oxygen, whose concentration in the tissue we denote by $\mathcal{C}$. In equilibrium, the diffusion of oxygen is balanced by uptake within the tissue and so we write

$$
0=D \nabla^{2} \mathcal{C}-R(\mathcal{C})
$$

Here $D$ is the diffusion coefficient of oxygen and $R(\mathcal{C})$ is an uptake term that represents the oxygen used by the tissue in, for example, respiration. Uptake of oxygen in tissue is typically modelled by a Michaelis-Menten term (Keener and Sneyd, 1998)

$$
R(\mathcal{C})=\frac{V_{m} \mathcal{C}}{\mathcal{C}+k_{m}}
$$

where $V_{m}$ and $k_{m}$ are constants representing the maximal uptake rate of oxygen, and the oxygen concentration at which the uptake rate falls to $V_{m} / 2$, respectively.

As the system must be solved on a deformable domain, we also map the governing equation for oxygen concentration (14) onto the Lagrangian domain,

$$
0=D \frac{1}{J} \nabla_{\mathbf{X}} \cdot\left[J \mathbf{F}^{-\mathrm{T}} \mathbf{F}^{-1}\left(\nabla_{\mathbf{X}} \mathcal{C}\right)\right]-R(\mathcal{C})
$$

where the relations between the Eulerian and Lagrangian description of the gradient of a scalar field and divergence of a vector field, (see Ogden, 1972, for example), have been used.

Recall that the $N$ capillaries are located at positions $R_{\text {cap }}^{(i)}=(i-1 / 2) H_{\text {cap }}(i=1 \ldots N)$ (see $\left.\S 2.1\right)$. For the oxygen model, we impose boundary conditions at $R=0, R=H$, and on each capillary $R=R_{\text {cap }}^{(i)}$. At the origin $(R=0)$ we have symmetry, and we assume no oxygen flux out of the tissue boundary $(R=H)$. Thus,

$$
\left.\frac{\mathrm{d} \mathcal{C}}{\mathrm{d} R}\right|_{0}=0,\left.\quad \frac{\mathrm{d} \mathcal{C}}{\mathrm{d} R}\right|_{H}=0 .
$$

We allow each capillary to be either open or closed, depending upon the mechanical stress within the tissue at that point. Then,

$$
\begin{cases}\left.\mathcal{C}\right|_{R_{\text {cap }}^{(i)}}=\mathcal{C}_{\text {cap }}, & \text { if } i \text { th capillary open } \\ {[\mathcal{C}]_{R_{\text {cap }}^{(i)}}=0=\left[\frac{1}{f^{\prime}} \frac{\mathrm{d} \mathcal{C}}{\mathrm{d} R}\right]_{R_{\text {cap }}^{(i)}},} & \text { if } i \text { th capillary closed }\end{cases}
$$

for $i=1 \ldots N$, where $[\mathcal{C}]_{R}=\mathcal{C}_{R^{+}}-\mathcal{C}_{R^{-}}$denotes the jump at position $R$. Equation (18) states that the tissue oxygen concentration $\mathcal{C}$ is equal to the capillary oxygen concentration $\mathcal{C}_{c a p}$ if the capillary is open, whilst if the capillary is closed, the presence of the capillary has no effect on the oxygen concentration and it is removed from the computation.

We also define a critical oxygen concentration, $\mathcal{C}_{\text {crit }}$, below which the tissue is assumed to become ischemic and infarcted, causing exposure of intracellular FCD. This coupling between mechanics and oxygen concentration in the tissue is discussed in $\S 2.5$. 


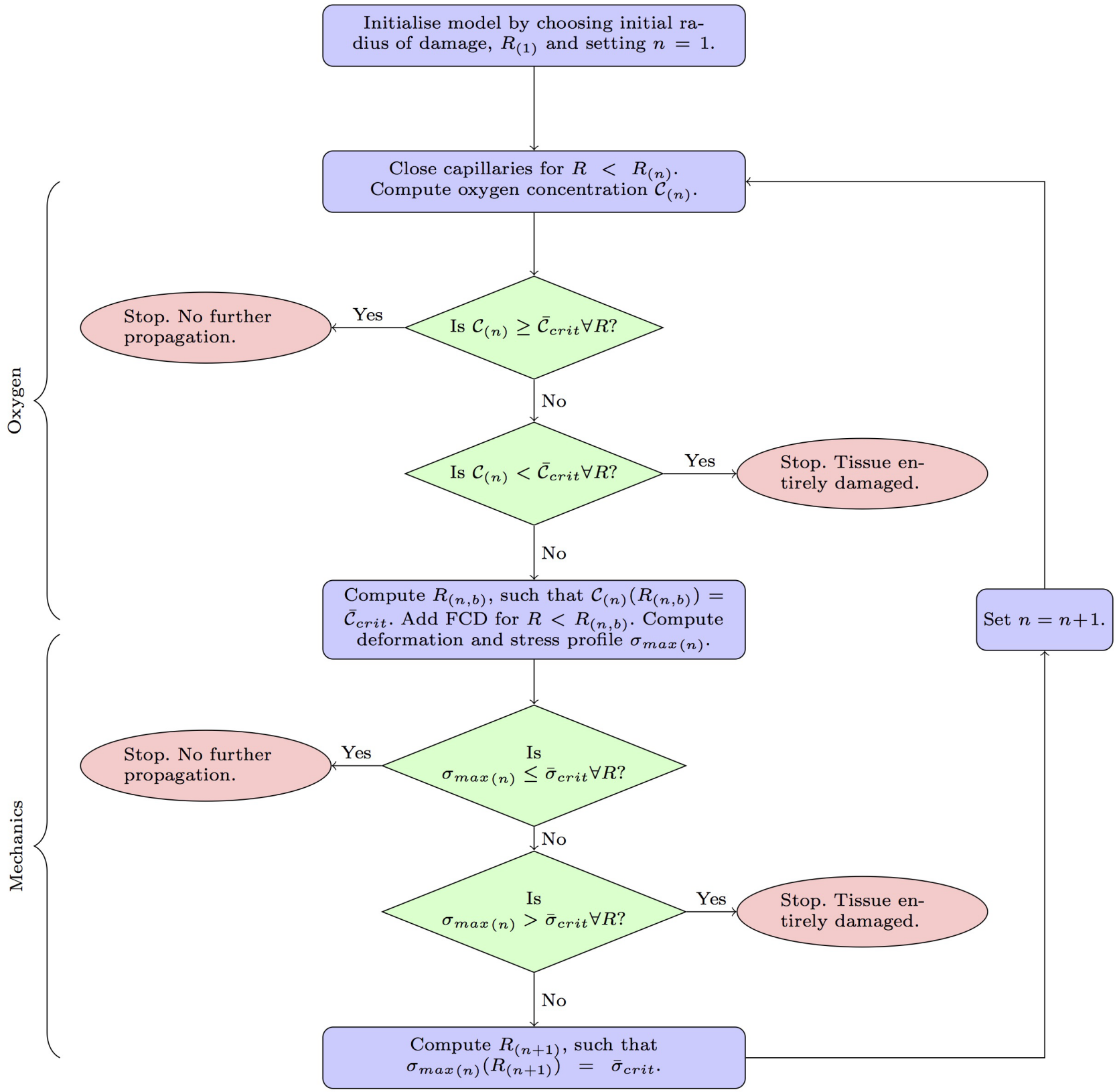

Fig. 2 A flow chart to show the stages of the coupled oxygen-mechanics damage model. The governing equations at the $n$th iteration are given in Appendix A.

\subsection{Non dimensionalisation}

We rescale the variables $f(R)$ and $\mathcal{C}(R)$, using the radius of the tissue region, $H$, as the natural length scale and the concentration of oxygen in capillaries, $\mathcal{C}_{\text {cap }}$, as the oxygen concentration scale. We therefore introduce the dimensionless variables

$$
\bar{R}=R / H, \quad \bar{f}(\bar{R})=f(R) / H, \quad \overline{\mathcal{C}}(\bar{R})=\mathcal{C}(R) / \mathcal{C}_{\text {cap }},
$$

where bars denote dimensionless quantities.

We choose the rescaling of pressure so that the dimensionless effective first Piola-Kirchhoff stress is defined by $\overline{\mathbf{S}}_{e}=\mathbf{S}_{e} /\left(\lambda_{s}+2 \mu_{s}\right)$ where $\lambda_{s}$ and $\mu_{s}$ are the Lamé constants obtained for any hyperelastic strain-energy function 
in the limit of small deformation. The other dimensionless parameters are defined as follows:

$$
\begin{aligned}
\bar{V}_{m} & =\frac{H^{2} V_{m}}{D}, & \bar{k}_{m} & =\frac{k_{m}}{\mathcal{C}_{\text {cap }}}, \\
\bar{\sigma}_{\text {crit }} & =\frac{\sigma_{\text {crit }}}{\lambda_{s}+2 \mu_{s}}, & \bar{c}^{*} & =\frac{R T c^{*}}{\lambda_{s}+2 \mu_{s}}, \\
\overline{\mathcal{C}}_{\text {crit }} & =\frac{\mathcal{C}_{\text {crit }}}{\mathcal{C}_{\text {cap }}} . & \bar{c}_{0}^{(f)}(R) & =\frac{R T c_{0}^{(f)}(R)}{\lambda_{s}+2 \mu_{s}} .
\end{aligned}
$$

Henceforth, we drop the bars from dimensionless variables but keep them on dimensionless parameters. For our study, the important dimensionless parameter governing the process is the critical stress $\sigma_{\text {crit }}$ at which a capillary will collapse as most of the other parameters are well characterised (see Table 1 ). The values of $\sigma_{\text {crit }}$ are not easily accessible and further complicated by the blood flow regulation in the brain; we consider a possible range of $0-10 \mathrm{mmHg}$ corresponding to a range for $\bar{\sigma}_{\text {crit }}$ between 0 and 1 . The governing equation for the oxygen concentration (14), with the Michaelis-Menten uptake term written explicitly, becomes

$$
\frac{1}{J} \nabla_{\mathbf{X}} \cdot\left[J \mathbf{F}^{-\mathrm{T}} \mathbf{F}^{-1}\left(\nabla_{\mathbf{X}} \mathcal{C}\right)\right]=\frac{\bar{V}_{m} \mathcal{C}}{\mathcal{C}+\bar{k}_{m}}
$$

while the Cauchy equation (10) becomes

$$
\nabla_{\mathbf{X}} \cdot \mathbf{S}_{e}=\nabla_{\mathbf{X}} \cdot\left(J \mathbf{F}^{-\mathrm{T}}\left[c^{* 2}+\left(\frac{\phi_{0}^{w} c_{0}^{(f)}}{J-1+\phi_{0}^{w}}\right)^{2}\right]^{1 / 2}\right)
$$

The dimensionless boundary conditions are given for completeness in Appendix A.

\subsection{Iterative approach for propagation}

We prescribe an initial radius of damage, $R_{(1)}$, representing the region of tissue within which blood flow is restricted following an injury, e.g. a stroke. The central question of interest is: how far can damage propagate without any additional imposed damage?

We impose damage by closing all capillaries in the region $R<R_{(1)}$. We then solve for the oxygen distribution (§2.3) within the tissue (subject to these capillaries being closed). From the oxygen distribution following damage, it is possible to determine the region within which oxygen levels fall below the critical oxygen threshold $\overline{\mathcal{C}}_{c r i t}$. In this hypoxic tissue, we impose an FCD (since the cells in this region are assumed to have died, and, hence, to have exposed their intracellular FCD). We then solve for the deformation of the tissue that is caused by this FCD distribution ( $\S 2.2)$.

If there is some radius $R_{(2)}>R_{(1)}$, such that the maximum compressive stress $\sigma_{\max }$ exceeds the critical threshold $\bar{\sigma}_{\text {crit }}$ for all $R<R_{(2)}$ then we assume that the blood flow in this region is restricted and the tissue is ischemic. We then define $R_{(2)}$ to be the new damage radius. (If $\sigma_{\text {max }}<\bar{\sigma}_{\text {crit }}$ for all $R>R_{(1)}$ then we set $R_{(2)}=R_{(1)}$ : damage does not propagate.)

In proceeding to the next iteration we note that, since $\sigma_{\max }>\bar{\sigma}_{\text {crit }}$ within $R<R_{(2)}$, all capillaries in $R<R_{(2)}$ are closed. We then iterate the above procedure, defining $R_{(n+1)}$ to be the radius of damage after $n$ iterations; $R_{(\infty)}$ is the final radius of damage, which is reached only when propagation has halted. Each iteration represents the time taken for ischemic tissue to die and expose its intracellular FCD. We call this iterative procedure the 'coupled oxygen-mechanics damage model'. A flow chart of this iterative process in shown in Figure 2, and the governing equations at the $n$th iteration are given in Appendix A.

\section{Critical oxygen concentration thresholds}

In this section, we focus on the coupling between oxygen and mechanics. Subsequently in $\S 4$ we focus on the mechanics.

\subsection{Parameter values}

Values of relevant parameters are shown in Table 1 . We emphasise that the inter-capillary distance $H_{\text {cap }}$ is fixed physiologically; the only geometrical parameter that we vary in this study is $H$, the size of the tissue region. 


\begin{tabular}{|c|c|c|c|c|c|}
\hline Parameter & Description & Value & Reference & Dimensionless & Value \\
\hline \multicolumn{6}{|l|}{ Geometry } \\
\hline $\begin{array}{l}H \\
H_{\text {cap }}\end{array}$ & $\begin{array}{l}\text { Radius of tissue region } \\
\text { Inter-capillary spacing }\end{array}$ & $\begin{array}{l}\ddagger \\
40 \mu \mathrm{m}\end{array}$ & Pardridge (1997) & $\begin{array}{l}\mathrm{n} / \mathrm{a} \\
\bar{H}_{c a p}=H_{\text {cap }} / H\end{array}$ & $\ddagger$ \\
\hline \multicolumn{6}{|c|}{ Mechanics } \\
\hline$\left(\lambda_{s}, \mu_{s}\right)$ & Elastic parameters & $\sim(500,250) \mathrm{Pa}$ & Cheng and Bilston (2007) & $\begin{array}{l}\bar{\lambda}_{s}=\lambda_{s} /\left(\lambda_{s}+2 \mu_{s}\right), \\
\overline{\mu_{s}}=\mu_{s} /\left(\lambda_{s}+2 \mu_{s}\right)\end{array}$ & $\begin{array}{l}0.50 \\
0.25\end{array}$ \\
\hline$c^{*}$ & Bathing ionic solutions & $300 \mathrm{mOsm}$ & Elkin et al. (2010) & $\overline{c^{*}}=R T c^{*} /\left(\lambda_{s}+2 \mu_{s}\right)$ & 750 \\
\hline$c_{0}^{(f)}$ & Damaged tissue FCD (ref. state) & $11.6 \mathrm{mOsm}$ & Lang et al. (2014) & $\bar{c}_{0}^{f}=R T c_{0}^{(f)} /\left(\lambda_{s}+2 \mu_{s}\right)$ & 40 \\
\hline$\phi_{0}^{w}$ & Ref state Brain tissue water content & & Go $(1997)$ & $\phi_{0}^{w}$ & 0.8 \\
\hline$\sigma_{\text {crit }}$ & Critical compressive stress & $*$ & & $\bar{\sigma}_{\text {crit }}=\sigma_{\text {crit }} /\left(\lambda_{s}+2 \mu_{s}\right)$ & * \\
\hline \multicolumn{6}{|l|}{ Oxygen } \\
\hline$D$ & Diffusion coefficient of $\mathrm{O}_{2}$ & $1.5 \times 10^{-9} \mathrm{~m}^{2} / \mathrm{s}$ & $\begin{array}{l}\text { Homer et al. (1983.) } \\
\text { Ganfield et al. (1970) }\end{array}$ & $\mathrm{n} / \mathrm{a}$ & \\
\hline $\mathcal{C}_{\text {c } a p}$ & Capillary $\mathrm{O}_{2}$ concentration & $0.05-0.1 \mathrm{mM}$ & & $\mathrm{n} / \mathrm{a}$ & \\
\hline $\mathcal{C}_{\text {crit }}$ & Critical $\mathrm{O}_{2}$ concentration & $0.02 \mathrm{mM}$ & ${ }^{\dagger \dagger}$ & $\overline{\mathcal{C}}_{\mathrm{crit}}=\mathcal{C}_{\mathrm{crit}} / \mathcal{C}_{\mathrm{c} a p}$ & $0.2-0.4$ \\
\hline$V_{m}$ & Maximal rate of $\mathrm{O}_{2}$ uptake & $0.1 \mathrm{mM} / \mathrm{s}$ & Secomb et al. (2000) & $\bar{V}_{m}=H^{2} V_{m} / D / \mathcal{C}_{\text {c } a p}$ & $\ddagger$ \\
\hline$k_{m}$ & Michaelis constant for $\mathrm{O}_{2}$ uptake & $5 \times 10^{-3} \mathrm{mM}$ & Secomb et al. (2000) & $k_{m}=k_{m} / \mathcal{C}_{\mathrm{c} a p}$ & 0.01 \\
\hline
\end{tabular}

for $\mathrm{O}_{2}$ uptake

$\dagger$ In the brain, the oxygen partial pressure of arterial blood (entering the brain) is $80-100 \mathrm{mmHg}$, and that of ventricular blood (leaving the brain) is $30-40 \mathrm{mmHg}$ (Secomb et al., 2000; Erecińska and Silver, 2001). Oxygen concentration $\mathcal{C}$ and oxygen partial pressure $P$ are related by $\mathcal{C}=\alpha P$, where $\alpha=1.29 \times 10^{-9} \mathrm{molO}_{2} \mathrm{mmHg}^{-1} \mathrm{ml}^{-1}$ is the oxygen solubility coefficient in water Atala and Lanza (2002). Therefore we assume that the capillary oxygen concentration is bounded by these values.

$\dagger^{\dagger}$ The critical value of oxygen partial pressure in the brain is the threshold below which we assume that tissue has insufficient oxygen and dies. Patients will be given treatment if their brain oxygen level falls below 20mmHg ( Narotam et al., 2009), and a mean oxygen tension in brain tissue of $19 \mathrm{mmHg}$ has been associated with poor outcome (Dhawan and DeGeorgia, 2012). Therefore we take $\mathcal{C}_{\text {crit }}=20 \mathrm{mmHg}$.

$\ddagger$ We shall consider several values of tissue radius, $H$, in our results. See figure captions for values.

Table 1 Parameter values

\subsection{Capillary closure and ischemia}

In Figure 3 we show oxygen concentration profiles through healthy tissue, for stages 0 (healthy tissue) and 1 (some capillaries closed) of the iteration process explained in $\S 2.5$. (For clarity of presentation, Figure 3 shows the concentration in a small region of tissue, with $H=5 H_{\text {cap }}$.) The grey region shows the range of physiological values for $\overline{\mathcal{C}}_{\text {crit }}$, as determined from a literature review (see Table 1 ).

(a)

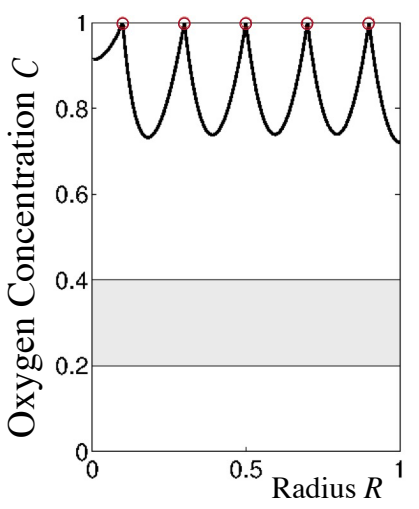

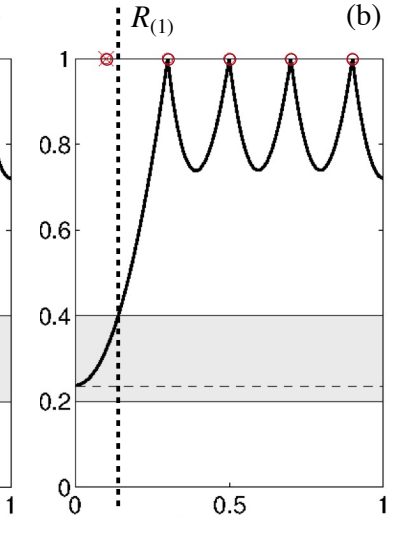

(b)

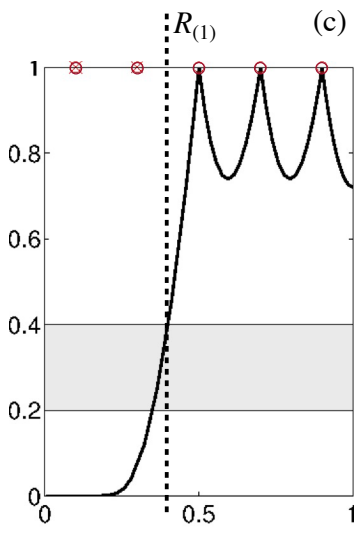

Fig. 3 Results for oxygen concentration $\mathcal{C}$, at steps 0 (healthy tissue) and 1 (capillaries situated at $R<R_{(1)}$ blocked) of the procedure described in Section 2.5. Profile of oxygen before a capillary is closed (a). The initial damage radius $R_{(1)}$ is chosen so that 1 capillary is closed in (b), and two capillaries are closed in (c). The grey region indicates the physiological range of the critical oxygen concentration, $\overline{\mathcal{C}}_{\text {crit }}$, for hypoxia. Open circles show the position of open capillaries, crossed circles the position of closed/blocked capillaries. Here $H=5 H_{\text {cap }}$ with all other parameter values as in Table 1 . Dashed lines denote the radii at which the oxygen concentration falls below critical.

We observe that for the parameters we consider: (a) when all capillaries are open the tissue has sufficient oxygen everywhere. If a single capillary is closed (case (b)), so that $0<R_{(1)}<H_{\text {cap }}$, a region of tissue will become hypoxic if the oxygen concentration $\mathcal{C}$ falls below the critical value $\overline{\mathcal{C}}_{\text {crit }}$ sufficiently close to the closed off region. This will occur provided that $\overline{\mathcal{C}}_{\text {crit }} \gtrsim 0.25$ (see dashed line in Figure $3(\mathrm{~b})$ ). However, if at least 2 capillaries are closed (case (c)) then some portion of the tissue will always become hypoxic, regardless of the value of $\overline{\mathcal{C}}_{\text {crit }}$. 
This general trend will be the same for other, physiologically relevant, parameter values. Indeed brain cells have a high oxygen requirement, and therefore oxygen cannot diffuse more than a few inter-capillary distances through the brain before it is consumed in respiration (Secomb et al., 2000). We conclude from this general pattern (as presented in Figure 3) that, provided an initial injury blocks blood supply to a region of tissue of size larger than $\approx 2 \times$ inter-capillary distance, then some portion of the tissue will become hypoxic. The swelling associated with this reduction of oxygen leads to the possibility of blood vessels collapsing in other parts of the tissue, leading to further ischemia and damage propagation.

\subsection{Relevance to the tissue length scale}

In Figure 3, we showed the oxygen concentration profile in a very small tissue region (with radius $5 \times$ the intercapillary distance, $\left.H_{\text {cap }}\right)$. In reality, we are more concerned with damage propagation on the length scale of a brain (centimetres, which will be many thousands of inter-capillary spacings.

We observe from Figure 3 that when a capillary is closed, whilst the oxygen concentration $\mathcal{C}$ will be below $\overline{\mathcal{C}}_{\text {crit }}$ for some distance away from the closed capillary, this distance is on the scale of the inter-capillary spacing $\bar{H}_{c a p}$. Therefore the precise value of the critical oxygen concentration $\overline{\mathcal{C}}_{\text {crit }}$ will only change the location of the infarcted tissue (and hence the location in which we impose an FCD), over a distance of the order of the inter-capillary distance. We conclude that, in the absence of autoregulation, the details of the oxygen diffusion and uptake may actually be relatively unimportant over the tissue length scale. This leads us to compare two models: the coupled oxygen-mechanics damage model described in $\S 2.5$, which accounts for the oxygen transport, and a simplified 'mechanics-only damage model', which does not explicitly include the details of oxygen transport. We describe the mechanics-only damage model next.

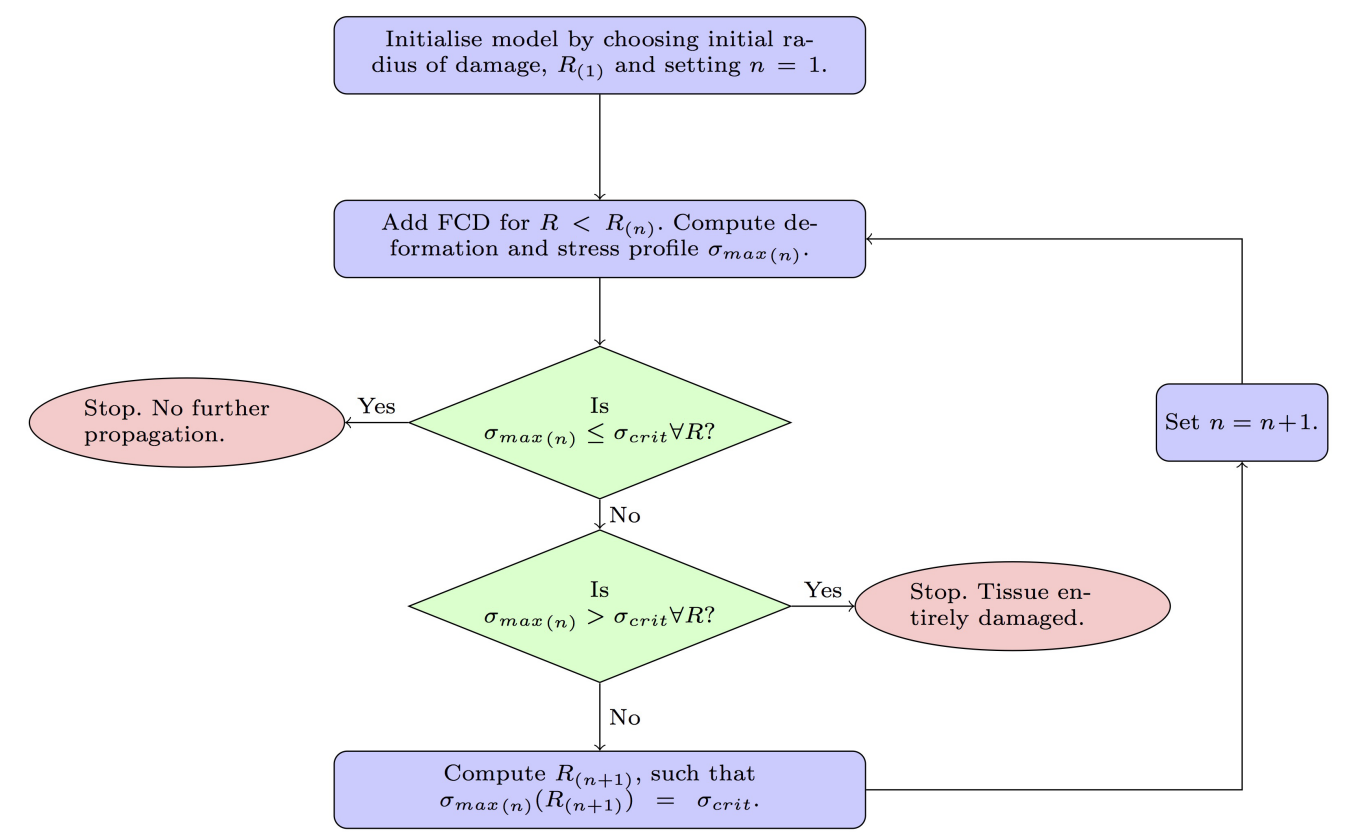

Fig. 4 A flow chart to show the stages of the mechanics-only damage model.

\subsubsection{The mechanics-only damage model}

In the mechanics-only model, we do not model the tissue oxygen concentration. Instead, we assume that any tissue experiencing a critical stress above $\bar{\sigma}_{\text {crit }}$ at iteration $n$ becomes hypoxic (and hence exposes its intracellular FCD) in iteration $n+1$. A flow chart of this iteration process is shown in Figure 4 ; the governing equations at the $n$th iteration are discussed in detail in Appendix B. 


\subsubsection{Comparison of mechanics-only damage and coupled oxygen-mechanics damage models}

In Figure 5, we compare the results of the coupled oxygen-mechanics damage model and the mechanics-only damage model, with no-displacement outer boundary conditions, eqn (11). We consider domains of radius 10 and 30 times the inter-capillary distance $H_{\text {cap }}$. The results are plotted with $R_{(n+1)}$ as a function of $R_{(n)}$ for each of the two models. We observe that both models display similar general trends. The major difference is that the results of the oxygen-mechanics model change in discrete steps (corresponding to the closure of individual capillaries), whilst the mechanics-only model yields a smooth curve (since FCD is being exposed continuously as the deformation increases, and not in response to capillary closure).

Note that the discrepancy between the two models decreases as further capillaries are added. This is because inclusion of the oxygen distribution increases resolution on the order of the dimensionless inter-capillary spacing $\bar{H}_{\text {cap }}=H_{\text {cap }} / H$; as the domain length $H$ increases, $\bar{H}_{c a p}$ decreases. This observation suggests that on domains of the size of primary interest in the brain (i.e. $H \approx 1 \mathrm{~cm}$ ), inclusion of the oxygen distribution will not noticeably affect the results. Therefore, we consider the mechanics-only damage model henceforth, since this will facilitate analytical progress. We emphasise that this simplified mechanics-only damage model implicitly assumes that closure of capillaries leads to hypoxia and cell death.

\section{Damage propagation}

We now investigate the conditions under which damage propagates using the mechanics-only damage model. In particular, we are interested in understanding how the critical stress at which we assume that capillaries become blocked, $\bar{\sigma}_{\text {crit }}$, together with the choice of boundary conditions at the outer surface, affect propagation. We consider two different boundary conditions on the outer surface: no displacement, to represent an intact skull, and no stress, to represent craniectomy; these conditions are expressed mathematically in (11). Comparing the results with these different boundary conditions allows us to understand the relative benefits of craniectomy versus no intervention.

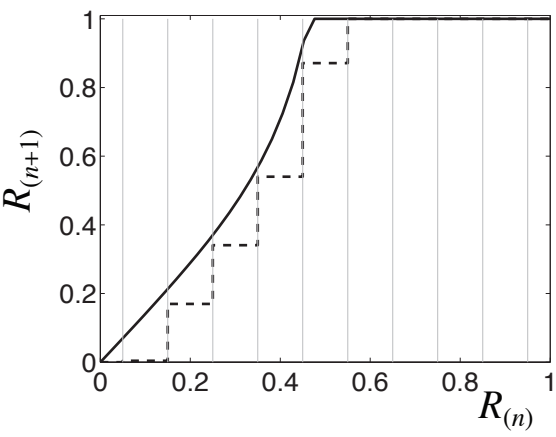

(a) $H=10 H_{\text {cap }}$

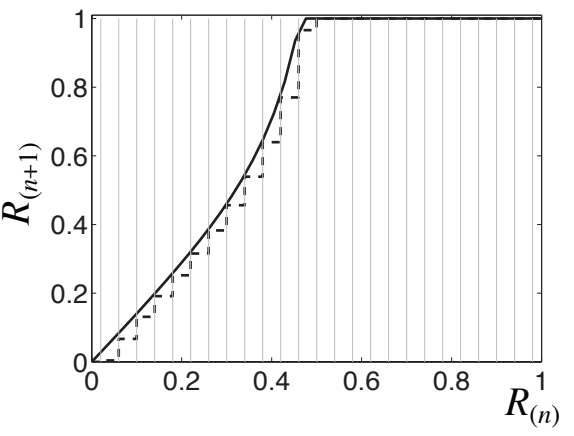

(b) $H=30 H_{\text {cap }}$

Fig. 5 A comparison of the new damage radius $R_{(n+1)}$ as a function of the previous damage radius $R_{(n)}$, computed using the coupled oxygen-mechanics damage model (dashed curves) and the reduced mechanics-only damage model (solid curves), for nodisplacement outer boundary conditions. Results are shown for two different domain sizes, $H$. The vertical grey lines indicate the positions of the capillaries. The coupled oxygen-mechanics damage model exhibits discrete steps, because the oxygen concentration profile remains unchanged until an additional capillary is closed. Here $\overline{\mathcal{C}}_{c r i t}=0.2$ with all other parameter values are as in Table 1.

In this section, we use a numerical approach to obtain results valid in finite elasticity. Subsequently, in $\S 5$, we shall consider an infinitesimal deformation model (i.e. linear elasticity) allowing us to make analytical progress; in particular, we are able to find an algebraic expression relating $R_{(n+1)}$ to $R_{(n)}$.

\subsection{Stress state caused by initial injury}

Figure 6 shows the spatial variation in the compressive stress and stretch in the radial and circumferential directions for two different sizes of the initial damaged region, $R_{(1)}$. Results are shown for each of the boundary 

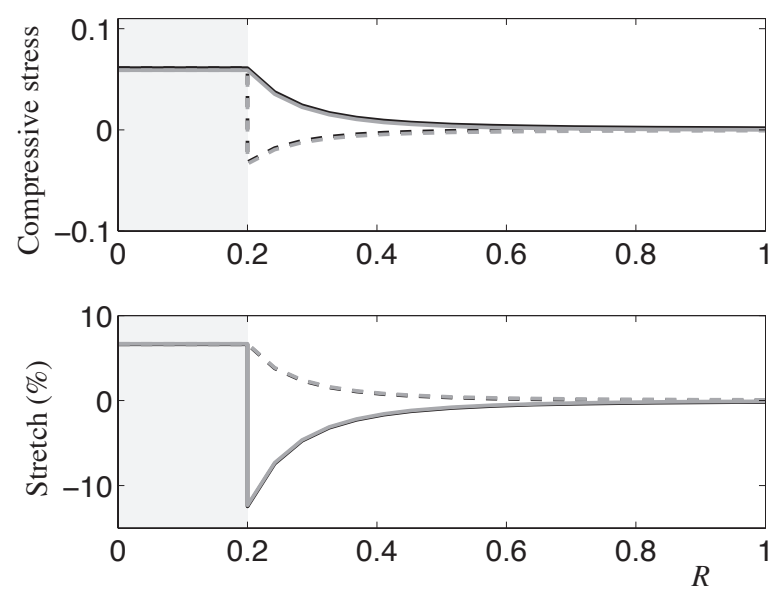

(a) Initial damage radius $R_{(1)}=0.2$
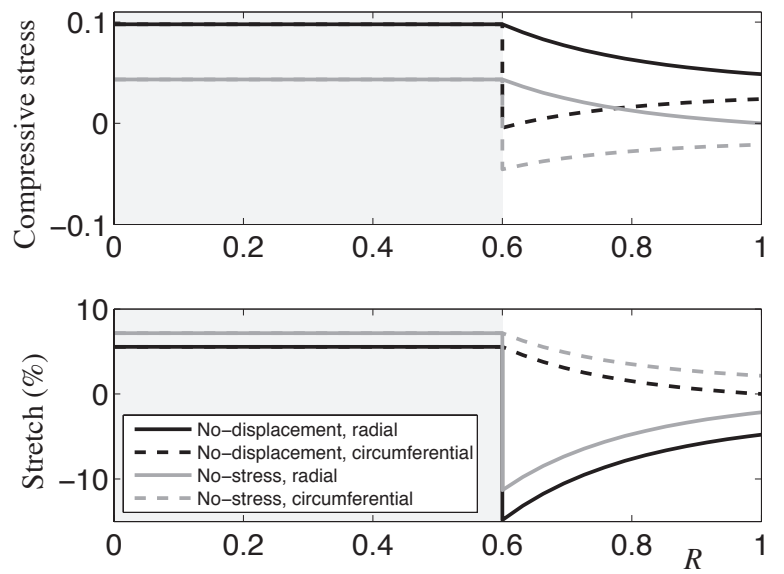

(b) Initial damage radius $R_{(1)}=0.6$

Fig. 6 Figures showing how a particular FCD distribution (grey shaded region) affects the compressive stress (upper) and stretch (lower) distributions within a spherically symmetric region of tissue for a single step of the propagation. Solid and dashed curves represent radial and circumferential values respectively: radial stretch is given by $\left(f^{\prime}-1\right)$ whilst circumferential stretch is given by $(f / R-1)$. The black curves show no-displacement boundary conditions, whilst the grey curves show no-stress boundary conditions. Here $H=1 \mathrm{~cm}$, with all other parameters as in Table 1.

conditions (11) and show that in both cases the compressive stress is greatest in the central infarcted region $\left(0<R<R_{(1)}\right)$. Furthermore, the radial and circumferential components of the stress are equal in this region. The radial compressive stress decreases with radius in the healthy tissue $\left(R_{(1)}<R<1\right)$, and is always positive (showing that the tissue is compressed radially). The circumferential compressive stress is negative in the healthy tissue $\left(R_{(1)}<R<1\right)$, meaning that the tissue is in circumferential tension. These observations correspond to the stretch profiles, which show that the tissue is always stretched in the circumferential direction, but is radially stretched in the central infarcted region and radially compressed in the surrounding region.

When a smaller region is damaged $\left(R_{(1)}=0.2\right)$, the stress and strain profiles are similar regardless of the boundary conditions. This is because the healthy region is sufficiently large that the stress decays through the healthy tissue and there is little deformation of the tissue at the outer boundary: the boundary conditions on the outer surface can have little effect on the behaviour of the tissue. However, if a larger region is damaged (for example, $R_{(1)}=0.6$ ), the radial stress within the healthy region is higher in the case of 'no-displacement' boundary conditions than in the case of 'no-stress' boundary conditions: the confinement caused by the boundary is felt by the tissue. 


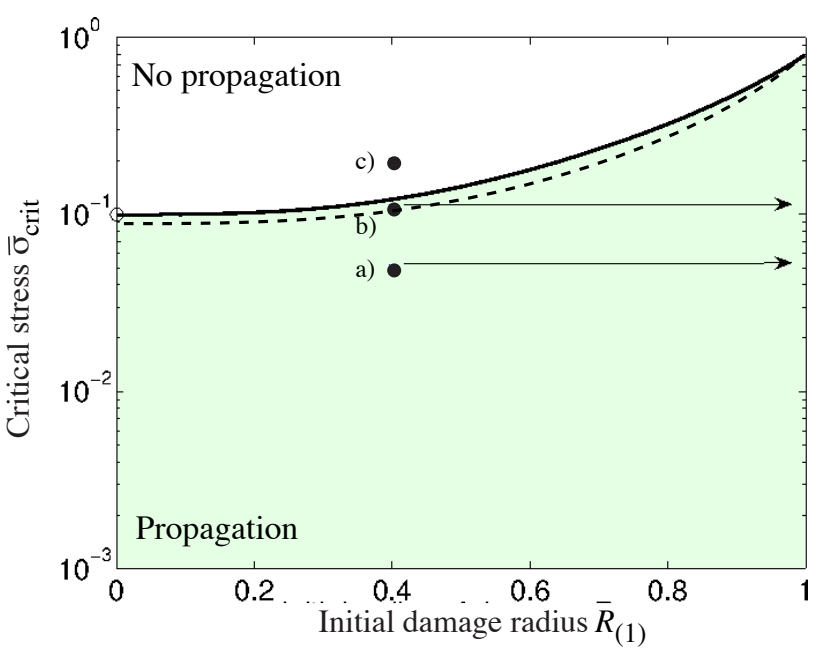

(a) No-displacement boundary condition

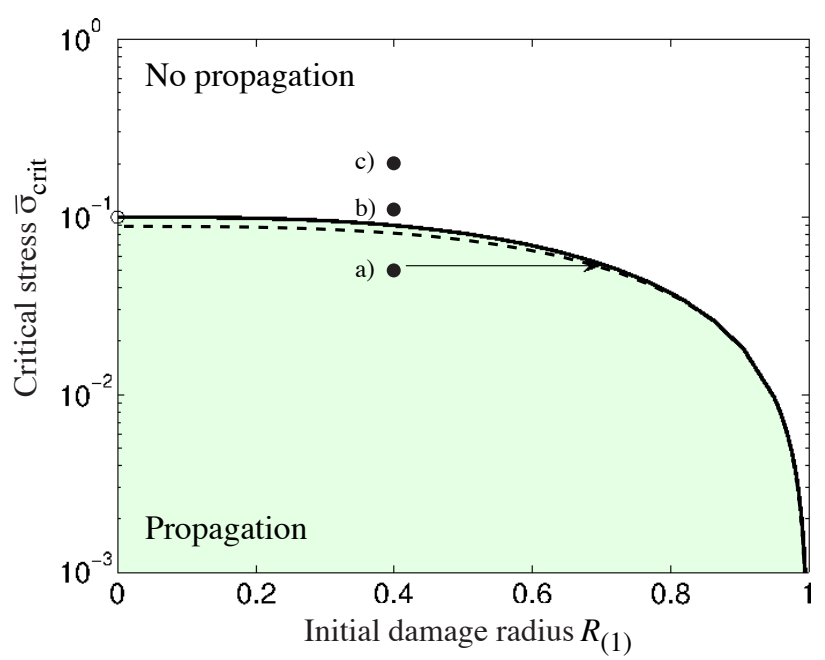

(b) No-stress boundary condition

Fig. 7 Regime diagram showing the regions of $\left(R_{(1)}, \bar{\sigma}_{\text {crit }}\right)$ parameter space for which damage will propagate. (Here, $R_{(1)}$ is the initial radius of damage while $\bar{\sigma}_{\text {crit }}$ is the critical compressive stress required to close capillaries.) Damage will propagate whenever parameters are chosen to be in the green region. The black solid curve dividing the regions is given by max $\left(-\hat{\mathbf{e}}_{r} \cdot \boldsymbol{\sigma} \cdot \hat{\mathbf{e}}_{r}\right)=\bar{\sigma}_{c r i t}$. Here $H=1 \mathrm{~cm}$ with all other parameters as in Table 1 . Points $(a),(b)$ and $(c)$ are chosen to highlight the different possible behaviours of the system (see text); they correspond to $R_{(1)}=0.4, \bar{\sigma}_{\text {crit }}=0.05,0.12$, and 0.2 , respectively. The circles at $R_{(1)}=0$ highlight that the limit $R_{(1)} \rightarrow 0$ is singular in the sense that an arbitrarily small amount of FCD will lead to a finite stress. The dashed lines correspond to the exact solution of the linear model given in Section 5 , showing reasonable qualitative agreement with the numerical model.

\subsection{Conditions for propagation}

We now consider the conditions under which damage may propagate through the tissue. First, we observe from the stress profiles shown in Figure 6 that the radial component of compressive stress tends always to be greater than or equal to the circumferential component. Furthermore, we observe that the radial compressive stress is constant within the infarcted region $\left(R<R_{(1)}\right)$, is continuous at $R=R_{(1)}$, and decreases monotonically within the healthy region $\left(R>R_{(1)}\right)$. Therefore, at any given iteration, damage will propagate into the healthy tissue if the maximum radial compressive stress is greater than the critical stress $\left(\max \left(-\hat{\mathbf{e}}_{r} \cdot \boldsymbol{\sigma} \cdot \hat{\mathbf{e}}_{r}\right)>\bar{\sigma}_{c r i t}\right)$, and will not propagate if the maximum radial compressive stress is less than or equal to the critical stress $\left(\max \left(-\hat{\mathbf{e}}_{r} \cdot \boldsymbol{\sigma} \cdot \hat{\mathbf{e}}_{r}\right) \leq \bar{\sigma}_{c r i t}\right)$.

Figure 7 shows a regime diagram summarising the behaviour of the system as the the critical stress $\bar{\sigma}_{\text {crit }}$ and the initial radius of damage $R_{(1)}$ change. This diagram illustrates the regions of parameter space within which damage will propagate. The curve separating the regions is $\max \left(-\hat{\mathbf{e}}_{r} \cdot \boldsymbol{\sigma} \cdot \hat{\mathbf{e}}_{r}\right)=\bar{\sigma}_{\text {crit }}$. The arrows illustrate that for a particular critical stress, damage will propagate outwards (away from $R=R_{(1)}$ ) until it reaches the damage radius at which $\max \left(-\hat{\mathbf{e}}_{r} \cdot \boldsymbol{\sigma} \cdot \hat{\mathbf{e}}_{r}\right)=\bar{\sigma}_{\text {crit }}$, if such a radius exists. If such a radius does not exist, propagation will continue until the entire tissue is damaged. The points $(a),(b)$ and $(c)$ in Figure 7 are chosen to highlight the difference in behaviour between the two different boundary condition. Starting from point (a), damage will initially propagate for both outer boundary conditions. However, in the case of 'no-displacement', the damage propagates to the boundary of the tissue; in the case of 'no-stress' propagation ceases with $R_{(\infty)}<1$. Similarly, starting from the point (b), damage will propagate only if the outer boundary condition is 'no-displacement'. Starting from point (c), the critical stress $\bar{\sigma}_{\text {crit }}$ is sufficiently high that the initial damage will not lead to propagation in either case.

The evolution of the compressive radial stress profiles through the tissue is shown in Figure 8 . The parameters $R_{(1)}$ and $\bar{\sigma}_{\text {crit }}$ are chosen to correspond to the points of parameter space $(a),(b)$ and $(c)$, marked by black circles in Figure 7. When $\bar{\sigma}_{\text {crit }}=0.05$ (Figure 8a), for $R_{(1)}=0.4$, the initial maximum compressive stress is greater than the critical stress threshold $\bar{\sigma}_{\text {crit }}$ and thus propagation occurs with either outer boundary condition. However, for no-stress boundary conditions, propagation halts once the radius of damage is approximately 0.7, whilst for no-displacement the entire tissue is finally damaged. When $\bar{\sigma}_{\text {crit }}=0.12$ (Figure $8 \mathrm{~b}$ ), no propagation occurs in the case of no-stress boundary conditions, whilst propagation does occur in the case of no-displacement boundary conditions. Finally, when $\bar{\sigma}_{\text {crit }}=0.2$ (Figure 8c), for $R_{(1)}=0.4$ the maximum compressive stress is below the critical stress threshold $\bar{\sigma}_{\text {crit }}$ for both sets of boundary conditions: no propagation occurs in either case. 


\section{No-displacement}

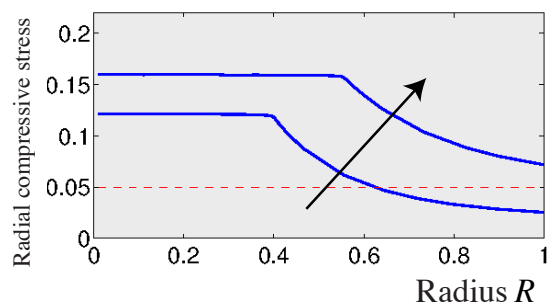

\section{No-stress}

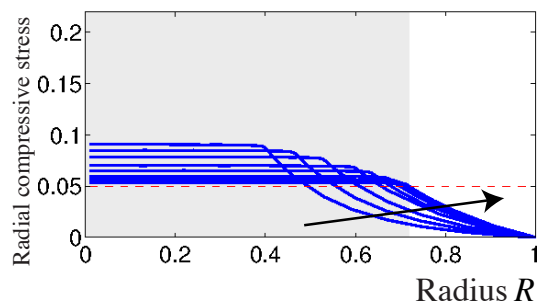

(a) $\bar{\sigma}_{\text {crit }}=0.05$
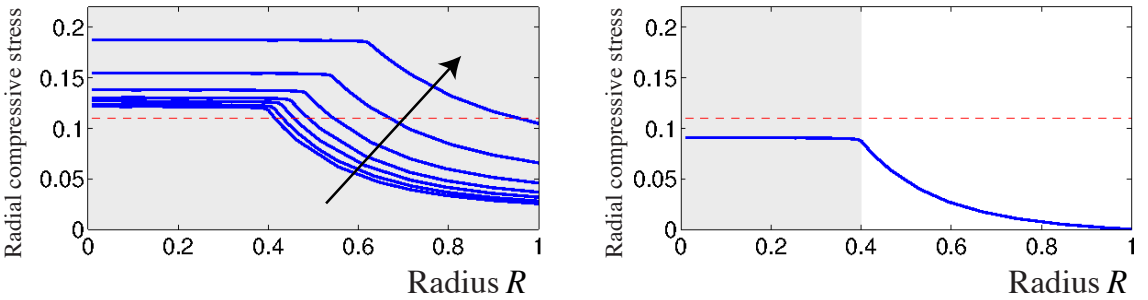

(b) $\bar{\sigma}_{\text {crit }}=0.12$
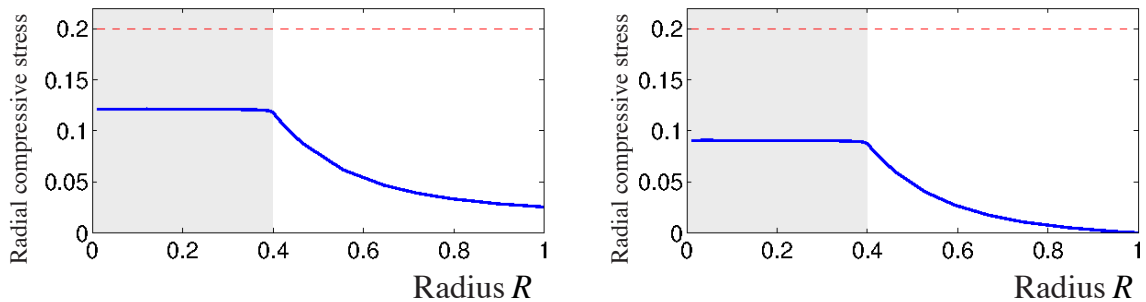

(c) $\bar{\sigma}_{\text {crit }}=0.2$

Fig. 8 Figure showing profiles of the radial compressive stress $\left(-\hat{\mathbf{e}}_{r} \cdot \boldsymbol{\sigma} \cdot \hat{\mathbf{e}}_{r}\right)$ through a sphere of tissue at successive iterations, for three different values of $\sigma_{\text {crit }}$. Each solid curve corresponds to a single iteration, with arrows in the direction of increasing number of iterations, and the dashed lines corresponding to $\bar{\sigma}_{\text {crit }}$. The grey shaded region shows the final radius of damage, $R_{(\infty)}$. No-displacement boundary condition is shown in the left column, and no-stress in the right column. Parameters: $R_{(1)}=0.4$, $H=1 \mathrm{~cm}$, all other parameters in Table 1.

Figure 9 shows the map from $R_{(n)}$ to $R_{(n+1)}$, and $R_{(\infty)}$ as a function of the initial damage radius $R_{(1)}$, for the three critical stresses $\left(\bar{\sigma}_{\text {crit }}=0.05,0.12,0.2\right)$ investigated in Figure 8. For no-displacement boundary conditions, we see that once propagation is initiated, then damage will propagate through the entire tissue. At each critical stress $\bar{\sigma}_{\text {crit }}$ there is some critical initial radius $R_{(1)}$ crit such that damage is initiated (and hence propagates through the whole tissue) when $R_{(1)}>R_{(1)}$ crit. However, in the case of no-stress boundary conditions, the larger two critical stresses $\left(\bar{\sigma}_{\text {crit }}=0.12,0.2\right)$ do not allow damage to propagate, whilst the lowest $\left(\bar{\sigma}_{\text {crit }}=0.05\right)$ does allow damage to propagate up to $R_{(\infty)} \approx 0.7$.

\subsection{Stress damage versus stretch damage}

Up to this point, we have only been concerned with the possibility that compressive stress may compress blood vessels, leading to ischemia and the propagation of damage. In this sense, our results indicate that craniectomy should always leads to a better outcome: the stress profiles we compute with 'no stress' outer boundary conditions (a craniectomy) are always lower than those with 'no displacement' boundary conditions, reducing the propagation of damage. However, in cohort studies of human patients, Cooper et al. (2011) observed that whilst a decompressive craniectomy reduces intracranial pressure, the procedure actually leads to more unfavourable outcomes for patients (such as death, vegetative state, or severe disability). They propose that this is because the craniectomy causes axonal stretch and leads to neural injury. 

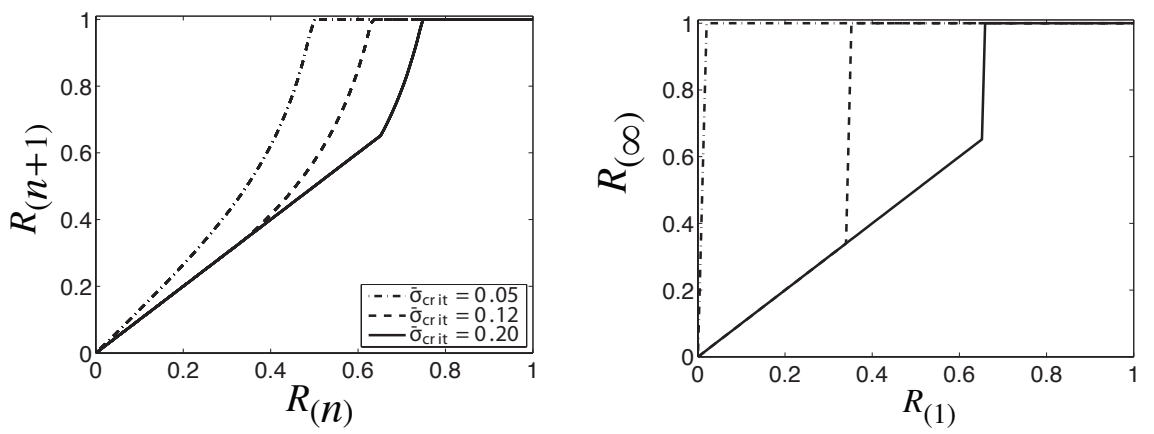

(a) No-displacement boundary condition
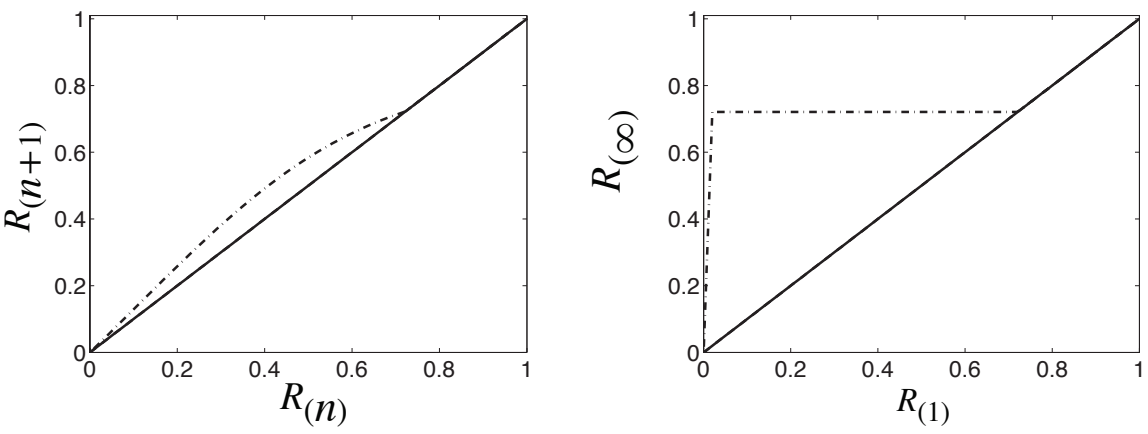

(b) No-stress boundary condition

Fig. 9 Map of $R_{(n)}$ to $R_{(n+1)}$ (left figures) and $R_{(1)}$ to $R_{(\infty)}$ (right figures). Dot-dashed curves correspond to $\bar{\sigma}_{c r i t}=0.05$, dashed curves to $\bar{\sigma}_{\text {crit }}=0.12$, and solid curves to $\bar{\sigma}_{c r i t}=0.2$. In (b), the solid and dashed curves overlap. Parameters: $H=1 \mathrm{~cm}$, all other parameters in Table 1.

The degree of axonal damage depends on both the magnitude and the rate of stretch (see Bain and Meaney, 2000; Tang-Schomer et al., 2010, for example). However, experimental studies indicate that, regardless of the strain rate, strains above $\approx 15 \%$ can lead to electrophysiological and structural damage to axons (Bain and Meaney, 2000). We therefore consider a second measure of damage, stretch damage, and determine how the extent of stretch damage depends on the outer boundary condition.

Stretch damage is believed to result directly from the stretch of axons; Figure 10 therefore shows the maximum stretch in the healthy tissue $\left(R>R_{(n)}\right)$ after successive iterations, as a function of the initial damaged radius $R_{(1)}$. We observe that the maximum stretch is greater for no-stress boundary conditions, for all values of the initial radius of damage $R_{(1)}$. This is as expected because the no-stress boundary conditions allow the tissue to stretch outwards. Our model therefore confirms that a craniectomy may not always lead to the beneficial consequences expected when studying propagation alone: instead, it may be necessary to consider a trade-off between preventing ischemic damage propagation and minimising stretch damage to axons.

\section{Iteration scheme for a linear elastic model}

We now further simplify the mechanics-only damage model by using governing equations valid for infinitesimally small deformations. The assumption of small deformations allows the governing equation (32) and boundary conditions (36), (38), (40) to be sufficiently simplified that analytical solutions for the displacement, stretch and stress may be obtained at each iteration. This simplification allows us to investigate analytically the importance of different model parameters and boundary conditions on the propagation of damage reported earlier. We are especially interested in the relations between the initial radius of damage, $R_{(1)}$ and the final damage radius $R_{(\infty)}$.

In Appendix $\mathrm{C}$ we present the governing equations for the infinitesimal deformation model. There we determine analytical expressions for the displacement and maximum compressive stress in terms of the initial damage radius, $R_{(1)}$. Furthermore, we show that for both no-displacement and no-stress boundary conditions there is an firstreturn map $\mathcal{F}$ such that $R_{(n+1)}=\mathcal{F}\left(R_{(n)}\right)$, (see equations (57) and (62), respectively). 


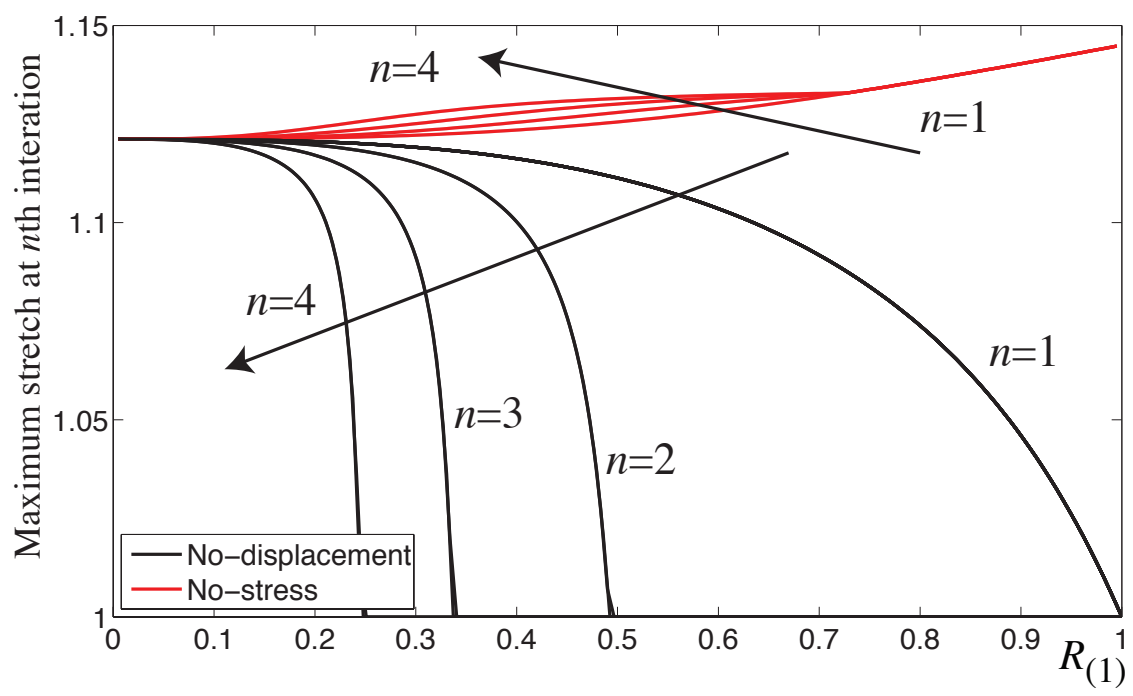

Fig. 10 Figure showing the maximum stretch in the healthy tissue $\left(R_{(n)}<R<1\right)$ at successive iterations, as a function of the initial damage radius $R_{(1)}$. To calculate the maximum stretch we take the local maximum of the radial stretch $\left(f_{(n)}^{\prime}-1\right)$ and circumferential stretch $\left(f_{(n)} / R-1\right)$. Black curves show results with no-displacement boundary conditions; red curves show the results with no-stress boundary conditions. In each case, arrows indicate the direction of successive iterations. Here, $H=1 \mathrm{~cm}$, $\bar{\sigma}_{\text {crit }}=0.05$ and all other parameters are as in Table 1.

\subsection{No-displacement outer boundary conditions}

When applying the no-displacement boundary conditions at the outer edge, we obtain the first-return map (57). We find that propagation is initiated provided that $R_{(1)}>r_{a}$ where

$$
r_{a}=\left(\frac{\alpha_{2} \bar{\sigma}_{c r i t}-4 \alpha_{1} \bar{\mu}_{s}}{3\left(\alpha_{1} \bar{\kappa}_{s}+\alpha_{3} \bar{\sigma}_{c r i t}\right)}\right)^{1 / 3},
$$

and the dimensionless constants $\alpha_{1}, \alpha_{2}$, and $\alpha_{3}$ are given in Appendix C.

Furthermore, the map (57) shows that if $R_{(n)}>r_{a}$ then $R_{(n+1)}>R_{(n)}$. We therefore conclude that propagation will continue until the entire tissue is damaged and the final damage radius $R_{(\infty)}$ is given by

$$
R_{(\infty)}= \begin{cases}R_{(1)}, & \text { if } R_{(1)} \leq r_{a} \\ 1, & \text { if } R_{(1)}>r_{a}\end{cases}
$$

This result agrees qualitatively with our earlier numerical simulations using finite elasticity as shown in Figure 7a. We conclude that whatever the value of $\bar{\sigma}_{\text {crit }}$. damage will propagate throughout the system provided that the size of the initial injury, $R_{(1)}$, is larger than a threshold value that depends on $\bar{\sigma}_{\text {crit }}$.

\subsection{No-stress outer boundary}

When applying a no-stress condition at the outer boundary, we obtain the map (62). This map is qualitatively different to that found in the case of no-displacement boundary conditions: if $R_{(n)}>r_{c}$, where

$$
r_{c}=\left(1-\frac{\bar{\sigma}_{c r i t}\left(\bar{\kappa}_{s} \alpha_{2}+4 \bar{\mu}_{s} \alpha_{3}\right)}{4 \bar{\mu}_{s}\left(\bar{\kappa}_{s} \alpha_{1}+\bar{\sigma}_{c r i t} \alpha_{3}\right)}\right)^{1 / 3},
$$

then $R_{(n+1)}=R_{(n)}$ and damage ceases to propagate.

We find that if the initial damage radius $R_{(1)}<r_{c}$, then damage will propagate until $R_{(n)}=r_{c}$. However if $R_{(1)} \geq r_{c}$ then no propagation will occur. Therefore, provided $r_{c}<1$, damage will not propagate through the entire tissue. This result agrees qualitatively with observations based on the finite deformation model as shown in Figure $7 \mathrm{~b}$. The final damage radius $R_{(\infty)}$ is given by

$$
R_{(\infty)}=\max \left\{R_{(1)}, r_{c}\right\} .
$$




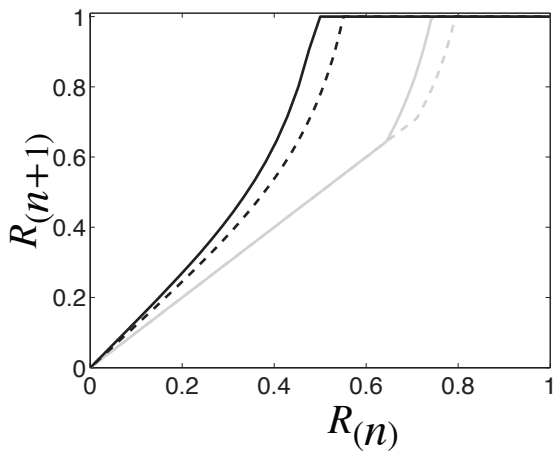

(a) No-displacement

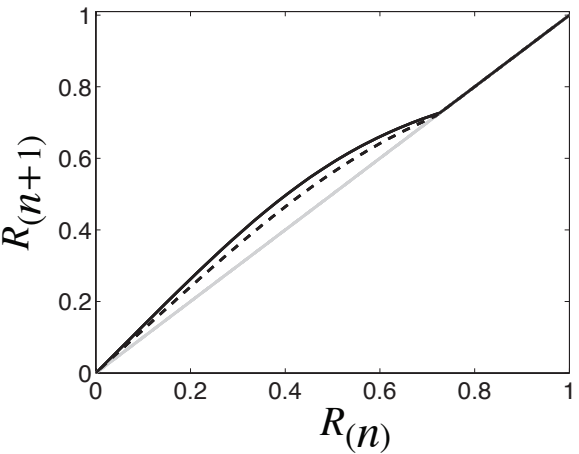

(b) No-stress

Fig. 11 Comparison of maps from $R_{(1)}$ to $R_{(2)}$ computed using the linear elasticity approach (dashed curves) and finite elasticity approach for the no-displacement case (a) or no-stress case (b). Parameters: $\bar{\sigma}_{c r i t}=0.05$ (grey) and $\bar{\sigma}_{c r i t}=0.2$ (black). In (b), the solid and dashed grey curves overlap. All other parameters are as in Table 1.

\subsection{Comparison of infinitesimal and finite results}

In $\S 4.2$ we investigated propagation effects using the mechanics-only damage iteration process and finite deformation elasticity. We showed that under no-displacement outer boundary conditions, if damage is initiated then the radius of damage will continue to propagate at each iteration until the entire tissue is damaged. However under no-stress outer boundary conditions, damage does not propagate through the tissue. In $\S 5.1$ and $\S 5.2$ we showed analytically that the same phenomenon is also observed in infinitesimal elasticity. In this final section, we present a quantitative comparison of the finite and infinitesimal models.

In Figure 11, we compare the first-return maps, the functions $\mathcal{F}$ such that $R_{(n+1)}=\mathcal{F}\left(R_{(n)}\right)$, that were calculated using the finite (see §4.2) and infinitesimal (equations (57), (62)) models. Results are shown for two different values of $\bar{\sigma}_{\text {crit }}$. We observe that the curves have similar shapes, although the curves computed for infinitesimal deformations consistently lie below those for finite deformations. This indicates that the maximum compressive stress in the tissue is smaller when computed using an infinitesimal rather than finite deformation model.

Figure 12 shows profiles of the radial compressive stress and radial stretch in the tissue, as calculated from both the finite and infinitesimal models. Profiles are shown for differing values of $\bar{c}_{0}^{f}$, which represents the FCD of infarcted tissue. The upper curves show results for $\bar{c}_{0 I}^{f}=40$, which is the value used throughout this work (see Table 1). We observe that, as expected, the compressive stress calculated using the infinitesimal deformation model falls slightly below that calculated using the finite deformation model. However, if $\bar{c}_{0}^{f}$ is reduced to 10 , so that smaller deformations are induced, the finite and infinitesimal results are indistinguishable on the scale of this plot, confirming that the finite deformation model does indeed reduce to the infinitesimal under small deformations.

\section{Discussion}

We have formulated a model to investigate the propagation of damage, caused by edema, through brain tissue. Our model couples a diffusion-uptake equation for oxygen, with a triphasic model for the tissue mechanics. Swelling is driven by exposure of intracellular FCD, assumed to be a consequence of low oxygen concentrations. It is important to comment on the generic features of our conceptual model and its many limitations.

We have used a simple model for diffusion and uptake of oxygen in the tissue, and assumed that capillaries act as an oxygen source, providing a fixed concentration when they are open. With this model, we have shown that explicitly modelling the microscopic oxygen concentration within the tissue is, in fact, unnecessary when considering macroscopic effects at the tissue scale. Indeed, qualitatively similar results may be obtained by assuming that the tissue becomes infarcted when the tissue experiences a compressive stress above the critical value, leading to exposure of intracellular FCD. Therefore, we conclude that the study of damage propagation does not require a detailed model of oxygen delivery even though oxygen regulation may be important (see below). Furthermore, we have used as a swelling mechanism the release of FCD following ischemia as it captures the observed rate of evolution of edema. 

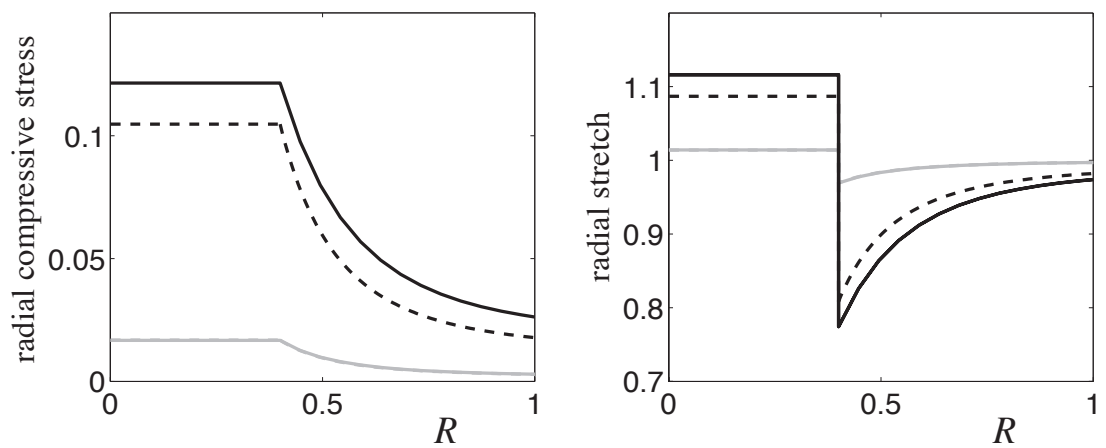

(a) No-displacement
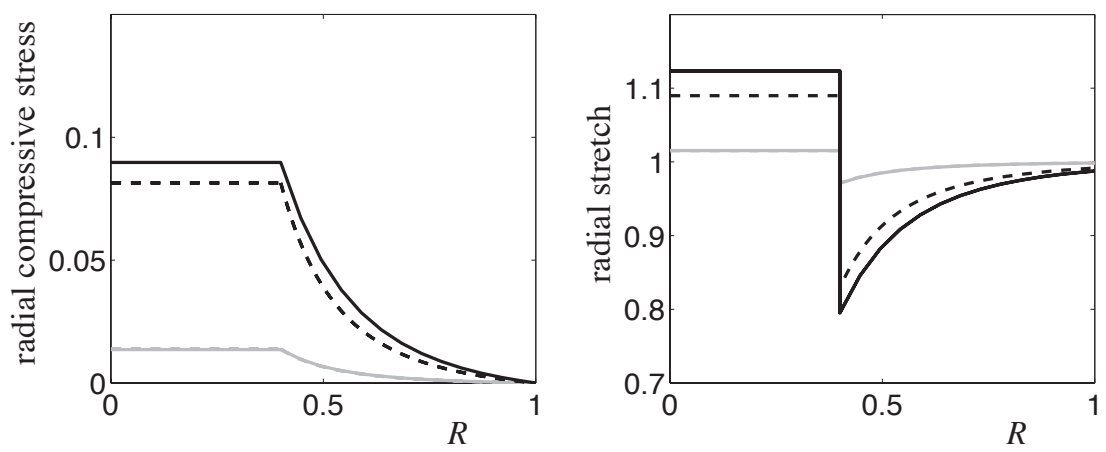

(b) No-stress

Fig. 12 Comparison of stress and stretch profiles using the computational finite approach (solid curves) and the analytical solution in the linear elasticity model (dashed curves). We show profiles for $\bar{c}_{0}^{f} I=10$ (grey) and $\bar{c}_{0}^{f}=40$ (black), with arrows in the direction of increasing $\bar{c}_{0}^{f}$. When $\bar{c}_{0}^{f}=10$, the finite and infinitesimal curves are indistinguishable on the scale of this plot.

The observation that damage propagation may cease with no-stress boundary conditions, yet continues throughout the entire tissue under no-displacement boundary conditions, is qualitatively consistent with the experimental results of Walberer et al. (2008). They showed that following an initial ischemic stroke the final size of damaged region is greater in rats who had not undergone a craniectomy. Our results thus provide a possible mechanical explanation for these experimental observations.

However, we have also shown that, despite the final volume of damage being smaller, the remaining tissue (and axons) experiences a greater stretch when a craniectomy is performed. We have neglected the mechanical effect of axons as reinforcing fibres in our analysis to provide an upper bound on the maximum stretch possible. In actuality, the fibre structures provided by both the neuronal network and vessel lining will resist stretch so that lower values of stretch should ultimately be expected. Nevertheless, the overall trend clearly establishes the trade-off between stresses and strains critical in the decisions related to intervention.

Another obvious limitation of our analysis is the simplified geometric setting considered. We have used a spherical geometry to model the (non-spherical) brain; an important additional consequence of this spherical geometry is that it forces us to consider spherical shells of oxygen sources, rather than individual point sources. It also restricts the analysis to the consideration of simple boundary conditions (completely open or completely closed), which is unrealistic since no craniectomy can fully remove the skull. Furthermore, the dura and other membranes sitting at the interface between the skull and brain would restrict the tissue expansion so that a craniectomy would not correspond precisely to stress free boundaries. Nevertheless, since we have established that oxygen variation between capillaries can be neglected in the process, the two choices of boundary conditions represent the two extreme situations for the increase of stresses and strains and should be used as bounds for possible behaviours rather than predictive data. Modifying this artificial geometry (and the associated simplifications) is a logical next step in the analysis of this problem. However, this will require a full finite-element analysis, either for a spherical brain and skull removing the assumption of spherical symmetry or, even more ambitiously, in a realistic skull geometry. 
In the analysis presented here, we have not included the ventricles. These sit at the centre of the brain and are a source of cerebrospinal fluid. Therefore, they both change the geometry and exert a pressure on the tissue at its interface with the interstitium (due to the pressure gradient between ventricles and sub-arachnoid space that is responsible for the overall fluid circulation). However, including both the restriction on the geometry (by considering a spherical shell rather than a sphere) and a small pressure of $1 \mathrm{mmHg}$, leads to negligible differences in the stress profiles in the outer region of the brain for both nonlinear and linear models (data not shown). We therefore conclude that our results on the propagation of damage do not depend on the presence of the ventricles.

We now comment on the assumptions for the levels of stress and oxygen that result in damage propagation. For oxygen we assumed that all open capillaries contain oxygen at a fixed concentration, and that when tissue oxygen concentration falls below a threshold $\mathcal{C}_{\text {crit }}$ the tissue becomes hypoxic and cells die. This assumption is a worst-case scenario as the blocking of blood vessels, ischemia, may be gradual so that a more complex model coupling oxygen availability to cerebral blood flow (for example Østergaard et al., 2013) may produce interesting results. For the mechanics of capillary closure, we assume that capillaries close when compressive stress in the tissue exceeds a certain level $\sigma_{\text {crit }}$. A more sophisticated model could be developed to couple the swelling of the tissue to capillary collapse. To our knowledge a model of this type does not exist for edema, but similar models have been developed, for example, to investigate capillary collapse in growing tumours (MacLaurin et al., 2012).

More importantly, we have neglected the ability of cerebral arteries to autoregulate blood flow (by dilating or contracting) in response to changes in perfusion pressure (the difference between arterial pressure and intracranial pressure). The relationship between cerebral blood flow and cerebral perfusion pressure, known as the autoregulation curve, is well documented in the biological literature (see Steiner and Andrews, 2006; Strandgaard et al., 1973, for example). In the first instance, a constitutive relation between local capillary pressure and local tissue pressure could be imposed (to allow cerebral blood flow to respond to changes in tissue pressure), based upon the autoregulation curve.

Our approach to studying the consequences of a craniectomy has been purely mechanical. There are multiple factors beyond mechanics that may affect clinical decisions as to whether a craniectomy is performed (e.g. risks of metabolic disturbances or infection (Fisher and Garcia, 1996)). Nevertheless, our model clearly indicates that propagation of damage can occur due to the interaction of swelling and blood flow.

\section{A The coupled mechanics-oxygen damage model}

In this Appendix, we state the governing equations at the $n$th iteration, for the coupled mechanics-oxygen damage model. At step $n=1$, we choose $R_{(1)}$ to be the initial radius of damage, and set $f_{(0)}(R) \equiv R$ everywhere (to represent that the tissue is initially undeformed).

Step a): Find the oxygen distribution $\mathcal{C}_{(n)}(R)$

We find the oxygen concentration profile at step $n, \mathcal{C}_{(n)}(R)$, by solving the steady diffusion-uptake equation for oxygen concentration (22). This equation can be rewritten, assuming spherical symmetry, as

$$
\frac{\mathrm{d}^{2} \mathcal{C}_{(n)}}{\mathrm{d} R^{2}}+\left(\frac{2 f_{(n-1)}^{\prime}}{f_{(n-1)}}-\frac{f_{(n-1)}^{\prime \prime}}{f_{(n-1)}^{\prime}}\right) \frac{\mathrm{d} \mathcal{C}_{(n)}}{\mathrm{d} R}=f_{(n-1)}^{\prime 2} \frac{\bar{V}_{m} \mathcal{C}_{(n)}}{\mathcal{C}_{(n)}+\bar{k}_{m}}
$$

along with boundary conditions,

$$
\begin{aligned}
& \left.\left.\frac{\left.\mathrm{d} \mathcal{C}_{(n)}\right|_{0}=0,}{\mathrm{~d} R}\right|_{1} \frac{\mathrm{d} \mathcal{C}_{(n)}}{\mathrm{d} R}\right|_{1}=0 \\
& \begin{cases}\left.\mathcal{C}_{(n)}\right|_{R_{c a p, i}}=1, & \text { if } R_{c a p, i}>R_{(n)}, \\
{\left[\mathcal{C}_{(n)}\right]_{R_{c a p, i}}=0=\left[\frac{1}{f_{n-1}^{\prime}} \frac{\mathrm{d} \mathcal{C}_{(n)}}{\mathrm{d} R}\right]_{R_{c a p, i}},} & \text { if } R_{c a p, i} \leq R_{(n)},\end{cases}
\end{aligned}
$$

where $R_{c a p, i}$ is the position of the $i$ th capillary ( $\left.\S 2.1\right)$. The last boundary condition in (30) is a mathematical representation of the assumption that if the capillaries are closed (i.e. if they are located within the damaged region $0<R<R(n)$ ) then they do not alter the flux or concentration of oxygen through them. However, if the capillaries are open $\left(R_{(n)}<R<1\right)$ then they fix the concentration at that point to be 1 . Note that the oxygen profile is solved on the deformed domain computed at the previous iteration (and hence $f_{(n-1)}$ appears in these governing equations). Matlab.

Equation (28), along with boundary conditions (29)-(30), are solved using the multipoint boundary value solver 'bvp5c' in 
Step b): Find the tissue deformation $f_{(n)}(R)$

We impose an $\operatorname{FCD} c_{0}^{(f)}{ }_{(n)}(R)$ wherever the oxygen concentration $\mathcal{C}_{(n)}$ falls below the critical value $\overline{\mathcal{C}}_{\text {crit }}$

$$
c_{0}^{(f)}(R)= \begin{cases}\overline{\mathcal{C}}_{0 I}^{f}, & \text { where } \mathcal{C}_{(n)}(R) \leq \overline{\mathcal{C}}_{\text {crit }} \\ 0, & \text { where } \mathcal{C}_{(n)}(R)>\overline{\mathcal{C}}_{\text {crit }}\end{cases}
$$

where $\bar{c}_{0 I}^{f}$ is a constant parameter representing the FCD of infarcted tissue.

If $\mathcal{C}_{(n)}>\overline{\mathcal{C}}_{\text {crit }}$ everywhere then the tissue receives sufficient oxygen everywhere. Then there is no propagation (and hence we cease the iterations). If $\mathcal{C}_{(n)}<\overline{\mathcal{C}}_{c r i t}$ everywhere then the entire tissue is ischemic and we set the radius of ischemic tissue, $R_{b(n)}=1$. Otherwise, let us define $R_{b(n)}$ as the value of $R$ such that $\mathcal{C}_{(n)}=\overline{\mathcal{C}}_{c r i t}{ }^{1}$.

Then, since the FCD is constant within each of the regions $0<R<R_{b(n)}$ and $R_{b(n)}<R<1$, the governing equation (23) can be solved with a constant FCD in each region. Boundary conditions must be applied at the interface between the damaged and healthy regions at $R=R_{b(n)}$ (equation (37)). In a spherically symmetric geometry the radial component of the governing equation $\nabla_{\mathbf{X}} \cdot \mathbf{S}_{e, n}=\mathbf{0}$ may be written,

$$
\begin{array}{r}
\frac{-f_{(n)}^{2} c^{(f)^{2}}}{R^{2}\left(J_{(n)}-\phi_{0}^{s}\right) \sqrt{c^{* 2}+c^{(f)^{2}}}}\left[\frac{f_{(n)}^{2} f_{(n)}^{\prime \prime}}{R^{2}}+\frac{2 f_{(n)} f_{(n)}^{\prime 2}}{R^{2}}-\frac{2 f_{(n)}^{2} f_{(n)}^{\prime}}{R^{3}}\right] \\
=\frac{\mathrm{d}\left(S_{R R(n)}\right)}{\mathrm{d} R}+\frac{2\left(S_{R R(n)}-S_{\Theta \Theta(n)}\right)}{R},
\end{array}
$$

where $c^{(f)}$ is given by $(7), J_{(n)}=f_{(n)}^{2} f_{(n)}^{\prime} / R^{2}$ is the volume change between the reference and deformed configurations, and $S_{R R(n)}$ and $S_{\Theta \Theta(n)}$ are the diagonal components of the effective Piola-Kirchhoff stress $\mathbf{S}_{e(n)}$. Note that in the outer region $\left(R_{b(n)}<R<1\right)$, the FCD $c^{(f)} \equiv 0$ and thus the LHS of equation (32) is zero.

Several strain energy functions have been utilised in the literature to represent brain tissue. For example, an Ogden type hyperelastic model was applied to brain tissue by García and Smith (2009) to model infusion tests, whilst the Fung model was used by Elkin et al. (2010) and Lang et al. (2014) to model the equilibrium behaviour of brain tissue slices in solution baths of differing concentrations. Lang (2014) investigated the behaviour of several of these strain energy functions and showed that, for moderate homogeneous swelling (bulk volume changes of up to 50\%), the neo-Hookean, Fung and Ogden models give quantitatively similar behaviour for parameters relevant to the brain.

Therefore, for simplicity we consider a neo-Hookean strain energy (see Ogden, 1984, for example). The components $S_{R R}$ and $S_{\Theta \Theta}$ required for the stress balance (32) may be written as functions of the radial deformation $f(R)$ :

$$
\begin{aligned}
& S_{R R(n)}=\frac{1}{f_{(n)}^{\prime}}\left[\bar{\mu}_{s}\left(f_{(n)}^{\prime 2}-1\right)+\bar{\lambda}_{s} J_{(n)}\left(J_{(n)}-1\right)\right], \\
& S_{\Theta \Theta(n)}=\frac{R}{f_{(n)}}\left[\bar{\mu}_{s}\left(\frac{f_{(n)}^{2}}{R^{2}}-1\right)+\bar{\lambda}_{s} J_{(n)}\left(J_{(n)}-1\right)\right] .
\end{aligned}
$$

The Cauchy stress $\boldsymbol{\sigma}_{(n)}$ is defined as,

$$
\boldsymbol{\sigma}_{(n)}=\boldsymbol{\sigma}_{e_{(n)}}-\left[\sqrt{\bar{c}^{* 2}+\left(\frac{\phi_{0}^{w} c_{0}^{(f)}(n)}{J_{(n)}-1+\phi_{0}^{w}}\right)^{2}}-\bar{c}^{*}\right],
$$

where the effective Cauchy stress $\boldsymbol{\sigma}_{e, n}$ is related to the effective Piola-Kirchhoff stress $\mathbf{S}_{e(n)}$ by $\boldsymbol{\sigma}_{e(n)}=\left(1 / J_{(n)}\right) \mathbf{F}_{(n)} \cdot \mathbf{S}_{e(n)}$. Note from equation (35) that in the absence of $\operatorname{FCD}\left(c_{0}^{(f)}=0\right)$, the Cauchy and effective Cauchy stresses are equal.

The boundary conditions for $f_{(n)}$ consist of no displacement at the origin,

$$
\left.f_{(n)}\right|_{0}=0,
$$

along with continuity of displacement and radial stress at the interface between the damaged and non-damaged region $R=R_{b(n)}$,

$$
\left[f_{(n)}\right]_{R_{b(n)}}=0, \quad\left[\hat{\mathbf{e}}_{r} \cdot \boldsymbol{\sigma}_{(n)} \cdot \hat{\mathbf{e}}_{r}\right]_{R_{b(n)}}=0 .
$$

Note that if $R_{b(n)}=1$ (i.e. the entire tissue has insufficient oxygen, so that $c_{0}^{(f)}(R)=\bar{c}_{0}^{f} I$ everywhere), then this boundary condition (37) is unnecessary. At the outer boundary $R=1$ we impose either no displacement or no stress,

$$
\left.f_{(n)}\right|_{1}=1, \quad \text { or }\left.\quad \hat{\mathbf{e}}_{r} \cdot \boldsymbol{\sigma}_{(n)} \cdot \hat{\mathbf{e}}_{r}\right|_{1}=0 .
$$

The governing equation (32), along with boundary conditions (36)-(38), is solved using a multipoint boundary value solver, 'bvp5c', in Matlab.

\footnotetext{
1 In practice, there is only one such value (see Figure 3).
} 
Step c): Find the new damage radius, $R_{(n+1)}$

We now consider the maximum compressive stress $\sigma_{\max (n)}(R):=\max \left(-\left(\hat{\mathbf{e}}_{r} \cdot \boldsymbol{\sigma}_{(n)} \cdot \hat{\mathbf{e}}_{r}\right),-\left(\hat{\mathbf{e}}_{\theta} \cdot \boldsymbol{\sigma}_{(n)} \cdot \hat{\mathbf{e}}_{\theta}\right)\right)$. We shall show in $\S \mathrm{C} .2$ and $\S$ C.3 that $\sigma_{\max (n)}(R)$ is a monotonic decreasing function over $R_{(n)}<R<1$; thus there are three possible cases:

i) $\sigma_{\max (n)}<\bar{\sigma}_{\text {crit }}$ for all $R_{(n)}<R<1$ : the damage does not propagate further, and $R_{(n+1)}=R_{(n)}$.

ii) $\sigma_{\max (n)}=\bar{\sigma}_{\text {crit }}$ for some $R_{(n)}<R<1$ : we define $R_{(n+1)}$ such that $\left.\sigma_{\max (n)}\right|_{R_{(n+1)}}=\bar{\sigma}_{c r i t}$.

iii) $\sigma_{\max (n)}>\bar{\sigma}_{\text {crit }}$ for all $R_{(n)}<R<1$ : the entire tissue is damaged; we set $R_{(n+1)}=1$.

We repeat steps (a) to (c) for step $n+1$ and iterate. A flow chart summary of this process is shown in Figure 2 .

\section{B The mechanics-only damage model}

Initially, we impose some initial radius of damage, $R_{(1)}$, and in subsequent steps, we take the value of $R_{(n)}$ from the previous step. At step $n$, we impose an FCD,

$$
c_{0}^{(f)}(R)=\left\{\begin{array}{ll}
\bar{c}_{0 I}^{f}, & R<R_{(n)}, \\
0, & R \geq R_{(n)}
\end{array},\right.
$$

and solve equation (32) in each of the regions $0<R<R_{(n)}$ and $R_{(n)}<R<1$, along with the boundary conditions (36) at $R=0$, (38) at $R=1$, and at $R_{(n)}$ :

$$
\left[f_{(n)}\right]_{R_{(n)}}=0, \quad\left[\hat{\mathbf{e}}_{r} \cdot \boldsymbol{\sigma}_{(n)} \cdot \hat{\mathbf{e}}_{r}\right]_{R_{(n)}}=0,
$$

where the Cauchy stress $\boldsymbol{\sigma}_{(n)}$ is given by $(35)$. We then define the maximum compressive $\operatorname{stress}$ as $\sigma_{\max }(n)(R):=\max \left(-\left(\hat{\mathbf{e}}_{r}\right.\right.$. $\left.\left.\boldsymbol{\sigma}_{(n)} \cdot \hat{\mathbf{e}}_{r}\right),-\left(\hat{\mathbf{e}}_{\theta} \cdot \boldsymbol{\sigma}_{(n)} \cdot \hat{\mathbf{e}}_{\theta}\right)\right)$, and find $R_{(n+1)}$ such that $\left.\sigma_{\max (n)}\right|_{R_{(n+1)}}=\bar{\sigma}_{c r i t}$ as described in step (c) of the 'mechanics-oxygen' model (§2.5). A flow chart of the stages shown in Figure 4.

\section{Analytical solutions for infinitesimal deformations}

In this Appendix, we consider the mechanics-only damage model described in $\S 3.3$, with the finite deformation governing equations replaced by governing equations valid only for infinitesimally small deformations. In the infinitesimal framework, deformations are assumed to be small and thus the Eulerian $(r)$ and Lagrangian $(R)$ frames are the same to leading order.

\section{C.1 Inner and outer solutions}

At the $n$th iteration we have a particular damage radius $R_{(n)}$, such that $\bar{c}_{0}^{f}=\bar{c}_{0}^{f}$ for $0 \leq r \leq R_{(n)}$ (the inner region), and $\bar{c}_{0}^{f}=0$ for $R_{(n)}<r \leq 1$ (the outer region). Let us consider each of these regions separately. We define $u_{I(n)}(r), u_{O(n)}(r)$ as the radial displacement in the inner and outer regions respectively. In each region the linear strain tensor is defined $\mathbf{e}_{i(n)}=$ $\operatorname{diag}\left[\mathrm{d} u_{i(n)} / \mathrm{d} r, u_{i(n)} / r, u_{i(n)} / r\right]$, and the dilation as,

$$
e_{i(n)}=\operatorname{tr}\left(\mathbf{e}_{i(n)}\right)=\frac{1}{r^{2}} \frac{\partial}{\partial r}\left(r^{2} u_{i(n)}\right), \quad(i=I, O),
$$

We use the infinitesimal stress tensor for the effective Cauchy stress, so that $\boldsymbol{\sigma}_{e i(n)}=\bar{\lambda}_{s} e_{i(n)}+2 \bar{\mu}_{s} \mathbf{e}_{i(n)}$. In infinitesimal deformation elasticity, the osmotic term in the Cauchy stress tensor (12) may be linearised. Then,

$$
\boldsymbol{\sigma}_{i(n)}=\boldsymbol{\sigma}_{e i(n)}-\left[\sqrt{\bar{c}_{0}^{f}{ }^{2}+\bar{c}^{* 2}}-\bar{c}^{*}-\left(\frac{\bar{c}_{0}^{f}{ }^{2}}{\phi_{0}^{w} \sqrt{\bar{c}_{0}^{f^{2}{ }^{2}+\bar{c}^{* 2}}}}\right) e_{i(n)}\right] \mathbf{I},
$$

where $\bar{c}_{0 I}^{f}$ is constant in the inner region, and $\bar{c}_{0}^{f} O=0$ in the outer region.

In each region the stress satisfies $\nabla \cdot \boldsymbol{\sigma}_{i(n)}=0$. Substituting in the Cauchy stress, (42), we find that

$$
0=\frac{\partial}{\partial r}\left(\frac{1}{r^{2}} \frac{\partial}{\partial r}\left(r^{2} u_{i(n)}\right)\right)
$$

in both the inner and outer regions. The general solution of equation (43) is,

$$
u_{i(n)}=a_{i(n)} r+\frac{b_{i(n)}}{r^{2}},
$$

where $a_{i(n)}$ and $b_{i(n)}$ are constants that must be determined from the boundary conditions.

At the origin, we have a no-displacement boundary condition,

$$
\left.u_{I(n)}\right|_{0}=0,
$$


which immediately gives $b_{I(n)}=0$. At the boundary between the outer and inner region, both the radial component of stress and the displacement must be continuous. Thus,

$$
\left.u_{I(n)}\right|_{R_{(n)}}=\left.u_{O(n)}\right|_{R_{(n)}},\left.\quad \quad \quad \hat{\mathbf{e}}_{r} \cdot \boldsymbol{\sigma}_{i(n)}\right|_{R_{(n)}} \cdot \hat{\mathbf{e}}_{r}=\left.\hat{\mathbf{e}}_{r} \cdot \boldsymbol{\sigma}_{O(n)}\right|_{R_{(n)}} \cdot \hat{\mathbf{e}}_{r} .
$$

At the outer edge, we apply either no-displacement or no-stress,

$$
\left.u_{O(n)}\right|_{1}=0, \quad \text { or }\left.\quad \hat{\mathbf{e}}_{r} \cdot \boldsymbol{\sigma}_{O(n)}\right|_{1} \cdot \hat{\mathbf{e}}_{r}=0
$$

We may now substitute the general expression for the displacement (44) into the boundary conditions (45)-(47) to solve for the displacement. Once $u_{i(n)}$ is determined, it is a simple matter to determine $\boldsymbol{\sigma}_{i(n)}$ and hence the region of $r$ for which $\sigma>\sigma_{\text {crit }}$. We consider the cases of no-displacement and no-stress boundary conditions separately.

\section{C.2 No-displacement outer boundary (intact skull)}

We will first consider the no-displacement outer boundary condition $\left(\left.u_{O(n)}\right|_{1}=0\right)$. Using equation (44) and boundary conditions (45)-(47) we find that the solutions for the inner and outer displacement may be written,

$$
u_{I(n)}=A_{(n)}\left(\frac{1}{R_{(n)}^{3}}-1\right) r, \quad u_{O(n)}=A_{(n)}\left(\frac{1}{r^{2}}-r\right),
$$

where the value of $A_{(n)}$ depends on $R_{(n)}$ according to,

$$
A_{(n)}:=A\left(R_{(n)}\right)=\frac{\alpha_{1} R_{(n)}^{3}}{\alpha_{2}-3 R_{(n)}^{3} \alpha_{3}},
$$

where the constants $\alpha_{1}, \alpha_{2}, \alpha_{3}$ are given by,

$$
\begin{array}{ll}
\alpha_{1}=\sqrt{\bar{c}_{0}^{f}{ }^{2}+\bar{c}^{* 2}}-\bar{c}^{*}, & \alpha_{2}=3 \bar{\kappa}_{s}+4 \bar{\mu}_{s}+3 \alpha_{3}, \\
\alpha_{3}=\frac{\bar{c}_{0 I}^{f}}{\phi_{0}^{w} \sqrt{{\overline{c_{0}^{f}}}^{2}+\bar{c}^{* 2}}}, &
\end{array}
$$

and $\bar{\kappa}_{s}=\bar{\lambda}_{s}+2 / 3 \bar{\mu}_{s}$ is the dimensionless bulk modulus. Using the general form of the Cauchy stress tensor (42), we can also write the radial component of the Cauchy stress in each of the inner and outer regions,

$$
\begin{aligned}
\sigma_{I(n)} & =3 A_{(n)}\left(\bar{\kappa}_{s}+\alpha_{3}\right)\left(\frac{1}{R_{(n)}^{3}}-1\right)-\alpha_{1}, \\
\sigma_{O(n)} & =-A_{(n)}\left[3 \bar{\kappa}_{s}+\frac{4 \bar{\mu}_{s}}{r^{3}}\right] .
\end{aligned}
$$

The maximal compressive stress in the tissue is,

$$
\sigma_{\max (n)}(r)= \begin{cases}\alpha_{1}-3 A_{(n)}\left(\bar{\kappa}_{s}+\alpha_{3}\right)\left(\frac{1}{R_{(n)}^{3}}-1\right), & r \leq R_{(n)}, \\ A_{(n)}\left(3 \bar{\kappa}_{s}+\frac{4 \bar{\mu}_{s}}{r^{3}}\right), & r>R_{(n)} .\end{cases}
$$

Equation (54) shows that at every step of the iteration, the maximum compressive Cauchy stress is constant within the inner region $\left(0<r<R_{(n)}\right)$, and monotonic decreasing in the outer region $\left(R_{(n)}<r<1\right)$. This trend agrees well with the numerical results obtained in $\S 4.2$ (see Figure 8 ) for the finite elasticity case.

For some radius of damage $R_{(n)}$, we now consider the damage radius following one iteration, $R_{(n+1)}$. Following the procedure described in $\S 3.3$, there are three cases to consider. Firstly, if the maximal compressive stress in the tissue $\sigma_{\max (n)}(r)$ is below the critical stress $\bar{\sigma}_{\text {crit }}$ everywhere, then damage does not propagate and $R_{(n+1)}=R_{(n)} \forall n>0$. Secondly, if there is some value of $R_{(n+1)}$ such that $R_{(n)}<R_{(n+1)}<1$ and $\sigma_{\max (n)}\left(R_{(n+1)}\right)=\sigma_{\text {crit }}$, then the damage will propagate up to that value. Thirdly, if the compressive stress $\bar{\sigma}_{\text {crit }}$ exceeds the critical stress everywhere, then the entire tissue is damaged and hence $R_{(n+1)}=1$.

These three cases depend on the size of $R_{(n)}$, relative to two length scales $r_{a}$ and $r_{b}$,

$$
\begin{aligned}
& r_{a}=\left(\frac{\alpha_{2} \bar{\sigma}_{c r i t}-4 \alpha_{1} \bar{\mu}_{s}}{3\left(\alpha_{1} \bar{\kappa}_{s}+\alpha_{3} \bar{\sigma}_{c r i t}\right)}\right)^{1 / 3}, \\
& r_{b}=\left(\frac{\alpha_{2} \bar{\sigma}_{c r i t}}{4 \alpha_{1} \bar{\mu}_{s}+3\left(\alpha_{1} \bar{\kappa}_{s}+\alpha_{3} \bar{\sigma}_{c r i t}\right)}\right)^{1 / 3},
\end{aligned}
$$

the values of $R_{(n)}$ such that $\sigma_{\max (n)}\left(R_{(n)}\right)=\bar{\sigma}_{\text {crit }}$ and $\sigma_{\max (n)}(1)=\bar{\sigma}_{\text {crit }}$ respectively. 
These three cases can be summarised as,

$$
R_{(n+1)}= \begin{cases}R_{(n)}, & \text { for } R_{(n)} \leq r_{a} \\ \left(\frac{\left(r_{b}^{3}-r_{a}^{3}\right) R_{(n)}^{3}}{r_{b}^{3}\left(1-r_{a}^{3}\right)-R_{(n)}^{3}\left(1-r_{b}^{3}\right)}\right)^{1 / 3}, & \text { for } r_{a}<R_{(n)} \leq r_{b} \\ 1, & \text { for } R_{(n)}>r_{b}\end{cases}
$$

where the middle line in equation (57) is obtained by finding the value of $R_{(n+1)}$ such that $\sigma_{\max (n)}\left(R_{(n+1)}\right)=\bar{\sigma}_{c r i t}$ in equation (54).

Ultimately given some initial radius of damage, $R_{(1)}$, we are interested in finding $R_{(\infty)}$, the final radius of damage. We thus consider fixed points $R^{*}$ of the first-return map (57), by seeking solutions to (57) such that $R^{*}=R_{(n)}=R_{(n+1)} \forall n \geq 1$. It is trivial to observe that $R^{*}$ can take all values less than or equal to $r_{a}$, and also 1 . However, $R^{*}$ cannot take any values between $r^{a}$ and 1.

We now consider the final radius of damage, $R_{(\infty)}$, for a given initial radius of damage $R_{(1)}$. If $R_{(1)} \leq r_{a}$, then $R_{(1)}$ is a fixed point of the map; damage does not propagate and trivially $R_{(\infty)}=R_{(1)}$. Rearranging the expression (57), we find that if $r_{a}<R_{(n)} \leq r_{b}$ then $R_{(n+1)}>R_{(n)}$. Thus if $r_{a}<R_{(1)} \leq r_{b}$ then the damage radius $R_{(n)}$ will increase at each iteration until $R_{(n)}>r_{b}$, following which $R_{(n+1)}$ reaches a fixed point so that $R_{(\infty)}=1$; propagation is halted as the entire tissue is damaged. Finally, if $r_{b}<R_{(1)} \leq 1$ then $R_{(\infty)}=1$. These cases are summarised in equation (25).

\section{C.3 No-stress outer boundary (craniectomy)}

We now consider the no-stress outer boundary condition $\left(\left.\hat{\mathbf{e}}_{r} \cdot \boldsymbol{\sigma}_{O(n)}\right|_{1} \cdot \hat{\mathbf{e}}_{r}=0\right)$. Using equation (44) and boundary conditions (45)-(47) we find that the solutions for the inner and outer displacement may be written,

$$
u_{I(n)}=B_{(n)}\left(\frac{4 \bar{\mu}_{s}}{3 \bar{\kappa}_{s}}+\frac{1}{R_{(n)}^{3}}\right) r, \quad u_{O(n)}=B_{(n)}\left(\frac{4 \bar{\mu}_{s}}{3 \bar{\kappa}_{s}} r+\frac{1}{r^{2}}\right),
$$

where $B_{(n)}$ is a function of $R_{(n)}$ defined by,

$$
B_{(n)}:=B\left(R_{(n)}\right)=\frac{\bar{\kappa}_{s} \alpha_{1} R_{(n)}^{3}}{\bar{\kappa}_{s} \alpha_{2}+4 \bar{\mu}_{s} \alpha_{3} R_{(n)}^{3}},
$$

the constants $\alpha_{1}, \alpha_{2}, \alpha_{3}$ are given by equation (51).

Using the general form of the Cauchy stress tensor (42), we can also write the maximum compressive Cauchy stress in each of the inner and outer regions,

$$
\sigma_{\max (n)}(r)= \begin{cases}\alpha_{1}-\left(3 \bar{\kappa}_{s}+\alpha_{3}\right) B_{(n)}\left(\frac{4 \bar{\mu}_{s}}{3 \bar{\kappa}_{s}}+\frac{1}{R_{(n)}^{3}}\right), & r \leq R_{(n)}, \\ 4 \bar{\mu}_{s} B_{(n)}\left(\frac{1}{r^{3}}-1\right), & r>R_{(n)} .\end{cases}
$$

Again, we observe that $\sigma_{\max (n)}$ is constant for $r \leq R_{(n)}$ and monotonic decreasing for $r>R_{(n)}$, consistent with the stress profiles shown in Figure 8 for the finite elasticity case.

We now consider the relationship between the initial radius of damage, $R_{(1)}$, and the radius of damage after a single iteration, $R_{(2)}$. We find that there is a value $r_{c}$

$$
r_{c}=\left(1-\frac{\bar{\sigma}_{c r i t}\left(\bar{\kappa}_{s} \alpha_{2}+4 \bar{\mu}_{s} \alpha_{3}\right)}{4 \bar{\mu}_{s}\left(\bar{\kappa}_{s} \alpha_{1}+\bar{\sigma}_{c r i t} \alpha_{3}\right)}\right)^{1 / 3}
$$

such that $\sigma_{\max (n)}\left(R_{(1)}\right) \geq \bar{\sigma}_{c r i t}$ if $R_{(1)} \leq r_{c}$ and $\sigma_{\max (n)}\left(R_{(1)}\right)<\bar{\sigma}_{c r i t}$ if $R_{(1)}>r_{c}$. Note that $r_{c}<1$. We find an expression for $R_{(2)}$,

$$
R_{(n+1)}= \begin{cases}\left(\frac{4 \bar{\mu}_{s} B_{1}}{\bar{\sigma}_{c r i t}+4 \bar{\mu}_{s} B_{1}}\right)^{1 / 3}, & R_{(n)} \leq r_{c} \\ R_{(n)}, & R_{(n)}>r_{c}\end{cases}
$$

where the uppermost line in equation (62) is obtained by setting $\sigma_{\max (1)}\left(R_{(n+1)}\right)=\bar{\sigma}_{\text {crit }}$ in equation (60), and $r_{c}$ is the value of $R_{(1)}$ such that $\sigma_{\max (1)}\left(R_{(n)}\right)=\sigma_{\text {crit }}$. We observe that if $\bar{\sigma}_{c r i t}>4 \bar{\mu}_{s} \alpha_{1} / \alpha_{2}$ then there are no positive values for $r_{c}$. In this case no propagation can occur, since then $R_{(n+1)}=R_{(n)}$ for all positive $R_{(n)}$.

Finally, we consider the fixed points $R^{*}$ of the map (62), in order to identify the relationship between the initial damage radius $R_{(1)}$ and the final damage radius $R_{(\infty)}$. By seeking solutions of (62) for which $R^{*}=R_{(n+1)}=R_{(n)} \forall n \geq 1$, we find that there are no fixed points that take values less than $r_{c}$ (since $R_{(n+1)}>R_{(n)}$ if $R_{(n)}<r_{c}$ ), but observe that all radial values greater than $r_{c}$ are fixed points. Furthermore, rearrangement of equation (62) gives that $R_{(n+1)} \leq r_{c}$ if $R_{(n)} \leq r_{c}$, meaning that $R_{(\infty)}=r_{c}$ if $R_{(1)} \leq r_{c}$. These results are summarised in equation (27). 


\section{References}

A. Atala and R.P. Lanza. Methods of tissue engineering. Gulf Professional Publishing, 2002

A. C. Bain and D. F. Meaney. Tissue-level thresholds for axonal damage in an experimental model of central nervous system white matter injury. J. biomech. eng., 122(6):615-622, 2000.

M. Ben Amar and A. Goriely. Growth and instability in elastic tissues. Journal of the Mechanics and Physics of Solids, 53(10): 2284-2319, 2005.

S. Cheng and E. Bilston, L. Unconfined compression of white matter. J. Biomech., 40(1):117 - 124, 2007. ISSN 0021-9290. doi: 10.1016/j.jbiomech.2005.11.004.

D. J. Cooper, J. V. Rosenfeld, L. Murray, Y. M. Arabi, A. R. Davies, P. D’Urso, T. Kossmann, J. Ponsford, I. Seppelt, P. Reilly, et al. Decompressive craniectomy in diffuse traumatic brain injury. New Engl. J. Med., 364(16):1493-1502, 2011.

S. C. Cowin and S. B. Doty. Tissue mechanics. Springer, 2009.

V. Dhawan and M. DeGeorgia. Neurointensive care biophysiological monitoring. Journal of neurointerventional surgery, 4(6): 407-413, 2012.

F. G. Donnan. The theory of membrane equilibria. Chem. Rev., 1(1):73-90, 1924.

B. S. Elkin, M. A. Shaik, and B. Morrison III. Fixed negative charge and the Donnan effect: A description of the driving forces associated with brain tissue swelling and edema. Phil. Trans. Royal Soc. London A, 368(1912):585-603., 2010. doi: $10.1098 /$ rsta.2009.0223.

M. Erecińska and I. A. Silver. Tissue oxygen tension and brain sensitivity to hypoxia. Respiration physiology, 128(3):263-276, 2001.

J. Flechsenhar, J. Woitzik, K. Zweckberger, H. Amiri, W. Hacke, and E. Jüttler. Hemicraniectomy in the management of spaceoccupying ischemic stroke. Journal of Clinical Neuroscience, 20(1):6-12, 2013.

M. Fisher and J. H. Garcia. Evolving stroke and the ischemic penumbra. Neurology, 47(4):884-888, 1996.

R. Ganfield, P. Nair, and W. Whalen. Mass transfer, storage, and utilization of o2 in cat cerebral cortex. Am. J. Physiol., 219: 814821, 1970 .

J. J. García and J. H. Smith. A biphasic hyperelastic model for the analysis of fluid and mass transport in brain tissue. Ann. Biomed. Eng., 37:375-386, 2009. ISSN 0090-6964. doi: 10.1007/s10439-008-9610-0.

K. G. Go. The normal and pathological physiology of brain water Advances and technical standards in neurosurgery, 47-142, 1997

A. Goriely, J.A. W. van Dommelen, M. G. D. Geers, G. Holzapfel, J. Jayamohan, A. Jérusalem, S. Sivaloganathan, W. Squier, S. Waters, E. Kuhl. Mechanics of the Brain: Perspectives, Challenges, and Opportunities. http://link.springer.com/article/10.1007/s10237-015-0662-4

T. Gerriets, E. Stolz, M. Walberer, C. Müller, A. Kluge, A. Bachmann, M. Fisher, M. Kaps, and G. Bachmann. Noninvasive quantification of brain edema and the space-occupying effect in rat stroke models using magnetic resonance imaging. Stroke, 35:566-571, 2004. doi: 10.1161/01.STR.0000113692.38574.57.

S. Hatashita, J. T. Hoff, and S. M. Salamat. Ischemic brain edema and the osmotic gradient between blood and brain. J. Cereb. Blood Flow Metab., 8(4):525-559, 1988. doi: 10.1038/jcbfm.1988.96.

J. M. R. J. Huyghe and J. D. Janssen. Quadriphasic mechanics of swelling incompressible porous media. Int. J. Eng. Sci., 35(8): $793-802,1997$.

L. D. Homer, J. B. Shelton, and T. J. Williams. Diffusion of oxygen in slices of rat brain. Am. J. Physiol. Regul. Integr. Comp. Physiol., 244::R15-R22, 1983.

J. P. Keener and J. Sneyd. Mathematical physiology, volume 8. Springer, 1998.

W. M. Lai, J. S. Hou, and V. C. Mow. A triphasic theory for the swelling and deformation behaviors of articular cartilage. J. Biomech. Eng., 113(3):245-58, 1991.

G. E. Lang, P. S. Stewart, D. Vella, S. L. Waters, and A. Goriely. Is the Donnan effect sufficient to explain swelling in brain tissue slices? J. R. Soc. Interface, 11(96), 2014. doi: 10.1098/rsif.2014.0123.

G. E. Lang. Mechanics of swelling and damage in brain tissue: a theoretical approach. PhD thesis, University of Oxford, 2014.

J. MacLaurin, S. J. Chapman, G. W. Jones, and T. Roose. The buckling of capillaries in solid tumours. Proc. R. Soc. A, 468 (2148):4123-4145, 2012.

A. Marmarou, P. P. Fatouros, P. Barza, G. Portella, M. Yoshihara, O. Tsuji, T. Yamamoto, F. Laine, S. Signoretti, J. D. Ward, M. R. Bullock, and H. F. Young. Contribution of edema and cerebral blood volume to traumatic brain swelling in head-injured patients. J. Neurosurg., 93(2):183-193, 2000. doi: 10.3171/jns.2000.93.2.0183.

P. K. Narotam, J. F. Morrison, and N. Nathoo. Brain tissue oxygen monitoring in traumatic brain injury and major trauma: outcome analysis of a brain tissue oxygen-directed therapy: Clinical article. Journal of neurosurgery, 111(4):672-682, 2009.

R. W. Ogden. Large deformation isotropic elasticity-on the correlation of theory and experiment for incompressible rubberlike solids. Proceedings of the Royal Society of London. A. Mathematical and Physical Sciences, 326(1567):565-584, 1972.

R. W. Ogden. Non Linear Elastic Deformations. Ellis-Horwood, 1984.

L. Østergaard, S. Nørhøj Jespersen, K. Mouridsen, I. Klærke Mikkelsen, K. Ýr Jonsdottír, A. Tietze, J. U. Blicher, R. Aamand, N. Hjort, N. Kerting Iversen, et al. The role of the cerebral capillaries in acute ischemic stroke: the extended penumbra model. J. Cereb. Blood Flow Metab., 33(5):635-648, 2013.

W. M. Pardridge. Drug delivery to the brain. J. Cereb. Blood Flow Metab., 17(7):713-731, 1997.

M. C. Papadopoulos, S. Krishna, and A. Verkman Aquaporin water channels and brain edema. Mount Sinai J. Med., 69:242?248, 2002.

A. Raslan and A. Bhardwaj. Medical management of cerebral edema. Neurosurg. Focus, 22(5):1-12, 2007. doi: 10.3171/foc.2007.22.5.13.

T. W. Secomb, R. Hsu, N. B. Beamer, and B. M. Coull. Theoretical simulation of oxygen transport to brain by networks of microvessels: Effects of oxygen supply and demand on tissue hypoxia. Microcirculation, 7(4):237-247, 2000. ISSN 1549-8719. doi: 10.1111/j.1549-8719.2000.tb00124.x.

J. M. Simard, T. A. Kent, M. Chen, K. V. Tarasov, and V. Gerzanich. Brain oedema in focal ischaemia: molecular pathophysiology and theoretical implications. Lancet. Neurol., 6:258-68, 2007. doi: 10.1016/S1474-4422(07)70055-8.

L. A. Steiner and P. J. D. Andrews. Monitoring the injured brain: ICP and CBF. British Journal of Anaesthesia, 97(1):26-38, 2006. doi: $10.1093 / \mathrm{bja} / \mathrm{ael110.}$ 
S. Strandgaard, J. Olesen, E. Skinh øj, and N. A. Lassen. Autoregulation of brain circulation in severe arterial hypertension. $B r$ Med J., 1(5852):507-510., 1973.

D. N. Sun, W. Y. Gu, X. E. Guo, W. M. Lai, and V. C. Mow. A mixed finite element formulation of triphasic mechanoelectrochemical theory for charged, hydrated biological soft tissues. Int J Numer Meth Eng., 45:13751402, 1999.

M. D. Tang-Schomer, A. R. Patel, P. W. Baas, and D. H. Smith. Mechanical breaking of microtubules in axons during dynamic stretch injury underlies delayed elasticity, microtubule disassembly, and axon degeneration. The FASEB Journal, 24(5):14011410, 2010. doi: 10.1096/fj.09-142844

R. Thiex and S. E. Tsirka. Brain edema after intracerebral hemorrhage: mechanisms, treatment options, management strategies, and operative indications. Neurosurg. Focus, 22(5):1-7, 2007. doi: 10.3171/foc.2007.22.5.7.

M. Walberer, N. Ritschel, M. Nedelmann, K. Volk, C. Müller, M. Tschernatsch, E. Stolz, F. Blaes, G. Bachmann, and T. Gerriets. Aggravation of infarct formation by brain swelling in a large territorial stroke: a target for neuroprotection? J. Neurosurg., 109 (2):287-293, 2008. doi: 10.3171/JNS/2008/109/8/028. 